\title{
Search for isolated Galactic Centre stellar mass black holes in the IR and sub-mm range
}

\author{
P.B. Ivanov ${ }^{1 \star}$, V. N. Lukash ${ }^{1} \dagger$, S. V. Pilipenko ${ }^{1} \ddagger$ and M. S. Pshirkov ${ }^{2} \S$ \\ ${ }^{1}$ Astro Space Centre, P.N. Lebedev Physical Institute, 4/32 Profsoyuznaya Street, Moscow, 117810, Russia \\ 2 Sternberg Astronomical Institute, Moscow State University, 119992, Universitetskiy Prospekt, 13, Moscow, Russia
}

\begin{abstract}
We investigate a possibility to find an accreting isolated black hole (IBH) with mass $1-100 \mathrm{M}_{\odot}$ within Central Galactic Molecular Zone $(\mathrm{CMZ})$ in the submillimetre and IR spectral range with help of planned space observatories James Webb Space Telescope (JWST) and Millimetron (MM). We assume the spherical mode of accretion. We develop the simplest possible quantitative model of the formation of radiation spectrum in this range due to synchrotron emission and show that it fully agrees with the more complicated model of Ipser and Price 1982 for expected values of accretion rate.

If a substantial fraction of LIGO events was caused by merger of primordial black holes, the JWST would be able to find them provided that there is a cusp in distribution of dark matter in the Galaxy and that the accretion efficiency parameter $\lambda$ defined as the ratio of accretion rate onto IBH to its Bondi-Hoyle-Lyttleton value is larger than $\sim 10^{-2}$. A comparable amount of IBHs is also predicted by recent models of their formation due to stellar evolution. MM capabilities are hampered by the effect of confusion due to distant submillimetre galaxies, but it can also be used for such purposes if the confusion effect is properly dealt with. In case of efficient accretion with $\lambda \sim 1$, both instruments could detect IBHs even when their number densities are as low as $10^{-6} \mathrm{pc}^{-3}$.
\end{abstract}

\section{Key words:}

\footnotetext{
* E-mail: pbi20@cam.ac.uk (PBI)

† E-mail: lukash@asc.rssi.ru (VNL)

$\ddagger$ E-mail: sergey.f77@gmail.com (SVP)

$\S$ E-mail:pshirkov@sai.msu.ru (MSP, corresponding author)
} 


\section{INTRODUCTION}

Isolated stellar mass black holes (IBHs) can be either formed as a result of stellar evolution (see e.g. (Shapiro \& Teukolsky 1983), (Eldridge et al. 2011) and references therein) or be primordial (e.g. (Carr et al. 2016) and reference therein). Recently the interest in finding such black holes has grown significantly since the first detection of gravitational wave signal GW150914 originated from a merger of two $\sim 30 \mathrm{M}_{\odot}$ black holes followed by a number of several similar events. There are some arguments suggesting their primordial origin. For instance, (Sasaki et al. 2016) estimate dark matter fraction $f^{1}$ sufficient to explain the observed merger rate to be of the order of $10^{-3}$.

Unlike black holes in binary systems, IBH are expected to be quite dim. So far, several authors have investigated a possibility of their search through microlensing (e.g. (Bennett et al. 2002)), or observations in radio (e.g. (Gaggero et al. 2017), Maccarone (2005)), IR (e.g. (McDowell 1985), (Campana \& Pardi 1993)), optical (e.g. (Chisholm et al. 2003)) bands, Xrays (e.g. (Fujita et al. 1998), (Agol \& Kamionkowski 2002), (Fender et al. 2013), (Gaggero et al. 2017), Hektor et al. (2018)) and gamma rays (e.g. (Armitage \& Natarajan 1999)), see also references in those papers, with no confirmed candidates having yet been found.

Up to now only IBHs in the closest neighbourhood of the Sun or in the nearest molecular clouds have been considered as detectable sources, e.g. Fender et al. (2013). One of possible reason for the non-detection of IBHs would be small spatial volumes of these sites, which impedes finding objects with a low abundance. It is clear that much larger test volumes and new observational facilities with better sensitivities are needed for future attempts to observe IBHs.

A natural candidate for such a site would be a region close to the Galactic Centre, say, the central region with diameter $100-200$ pc. Firstly, the interstellar medium is sufficiently dense there, with the number density of hydrogen of the order of $10^{2} \mathrm{~cm}^{-3}$ much larger than the mean number density in our Galaxy $\sim 1 \mathrm{~cm}^{-3}$, thus, enhancing the accretion rate onto an IBH. Secondly, both recent calculations of spatial distribution of IBHs born as a result of stellar evolution (Tsuna et al. 2018) and the assumption that they are of primordial origin and should, accordingly, trace the distribution of dark matter suggest that they should concentrate near the Galactic Centre (GC), thus, pointing to this site as a natural place to

${ }^{1} f=\rho_{P B H} / \rho_{D M}$, where $\rho_{P B H}$ and $\rho_{D M}$ are mass densities of primordial black holes and dark matter, respectively. 
look for IBHs. Thirdly, the small angular size of this region implies that it can be easily surveyed during a reasonable observational time.

The search for IBHs in GC is obstructed by its large distance from Earth and large expected average velocities of IBHs with respect to the GC interstellar medium ${ }^{2}, v \sim$ $200 \mathrm{~km} \mathrm{~s}^{-1}$. However, the next decade will see the launches of two space observatories with unprecedented sensitivities in the IR and submillimetre range, James Webb Space Telescope (JWST, see e.g. https://www.jwst.nasa.gov/) and Millimetron (MM, see e.g. http://millimetron.ru/index.php/en/, (Kardashev et al. 2014) and (Ivanov et al. 2019)). Both instruments will reach the sensitivity of the order of $10^{-5} \mathrm{Jy}$, making it possible to detect extremely faint objects and, thus, suggesting them for the search of IBHs in GC.

It is the purpose of this paper to explore a possibility of detection of IBHs in GC with help of these observatories. Since the physics of accretion and formation of radiation spectrum in this waveband are rather poorly known, we confine ourselves to the simplest quantitative model of accretion and production of radiation in an accreting IBH. Namely, we assume that accretion proceeds through the Bondi-Hoyle-Lyttleton (BHL) mechanism at a rate

$$
\dot{M}=4 \pi \lambda \frac{G^{2} M^{2} \rho}{\left(v^{2}+c_{s}^{2}\right)^{3 / 2}},
$$

where $G$ is the gravitational constant, $M$ is the mass of a black hole travelling at velocity $v$ through interstellar medium of density $\rho$ and sound speed $c_{s}, \lambda$ is an efficiency factor, which takes into account that the accretion rate could be smaller than its 'canonical' BHL value, see e.g. Perna et al. (2003), Fender et al. (2013) and references therein.

We consider only the synchrotron mechanism of radiation emission in the accreting flow, because it is the only mechanism responsible for production of photons with frequencies in the IR and submillimetre band for expected values of electron number density, temperature and magnetic field. That an IBH must emit radiation due to this mechanism was first pointed out in the pioneering work (Shvartsman 1971). The first detailed theories of this process were proposed in (Shapiro 1973), (Bisnovatyi-Kogan \& Ruzmaikin 1976), although the former paper considered only the optically thin regime and neglected possible dissipation of magnetic field, while the latter dealt with a similar case of disc-like structures and also neglected the effect of dissipation. A detailed numerical calculation of emerging spectrum was done by (Ipser \& Price 1982), who considered gas heating due to dissipation of magnetic

\footnotetext{
2 The latter is due to the fact that accretion rate onto an IBH should be inversely proportional to the third power of $v$, see eq. (1).
} 
field, non-zero opacity due to synchrotron self-absorption and relativistic effects ${ }^{3}$. In these papers it was assumed that only thermal electrons contribute to radiation emission. Later, in (Beskin \& Karpov 2005) a possible contribution of non-thermal electrons was discussed, which changes the spectrum at frequencies higher than those available for observations with MM and JWST, and, therefore, is not important for our purposes as well as the process of Comptonization of synchrotron radiation.

We adopt an approach to calculation of the emerging spectrum, which closely follows (Ipser \& Price 1982), making, however, a set of additional simplifications, which reduce the calculations to a small number of simple quadratures. Namely, we assume that the magnetic field energy density is always proportional to gas gravitational energy density and that all quantities of interest are simple power law functions of distance from the black hole. Additionally, our model is fully Newtonian. There are two free parameters, the ratio of electron and proton temperatures and inner cut-off radius, which is chosen to be equal to the radius of the circular photon orbit for a non-rotating black hole. The ratio of temperatures is fixed by the requirement that our calculations give the result for the spectrum as close to the one obtained by (Ipser \& Price 1982) for particular values of black hole mass and accretion rate. We check that our model, remarkably, is able to reproduce quantitatively the results of (Ipser \& Price 1982) for a very wide range of black hole masses and sufficiently small accretion rates. This suggests that, when the accretion rate is small enough, the formation of spectrum does not depend on many details of more complicated models.

We use equation (1) to relate the accretion rate to parameters of Central Molecular Zone (CMZ) of GC assuming the gas number density unperturbed by IBHs to be equal to $10^{2} \mathrm{~cm}^{-3}$, see (Ferrière et al. 2007) and velocity dispersion $\sigma=200 \mathrm{~km} \mathrm{~s}^{-1}$ to be order of velocity dispersion of the bulge stars, see (Valenti et al. 2018). The sound speed of the medium is expected to be much smaller than $v$, and, therefore, is set to zero in (1). We treat the efficiency parameter $\lambda$ as a free one, and calculate the spectra for a range of $\lambda$ and black hole masses within the range $1 \mathrm{M}_{\odot} \leqslant M \leqslant 100 \mathrm{M}_{\odot}$ comparing them with the appropriate sensitivity curves for MM and JWST, taking into account a possible confusion between IBHs and background sources, like sub-mm IR galaxies. Note that all considered accretion rates are inside the region of applicability of our simplified model, see equation (12) below.

It is shown that MM and JWST are able to detect an IBH within CMZ travelling at a

3 See also (Ipser \& Price 1982) for a discussion of some other early works on the subject. 
typical speed $\sigma \sim 200 \mathrm{~km} \mathrm{~s}^{-1}$ when its mass is larger than $M_{\text {crit }} \approx 20 \mathrm{M}_{\odot}$ and $M_{\text {crit }} \approx 30 \mathrm{M}_{\odot}$, for MM and JWST, respectively and $\lambda \sim 1$. When $\lambda$ gets smaller $M_{\text {crit }}$ gets larger, and, for the largest considered $M_{\text {crit }}$, the minimal possible value of $\lambda \sim 0.1$ for both instruments.

The detection capabilities of MM are, however, hampered by the confusion limit, which results in $M_{\text {crit }} \approx 90 \mathrm{M}_{\odot}$ for $\sigma \approx 200 \mathrm{~km} \mathrm{~s}^{-1}$. Therefore, one must find a way to disentangle the contributions from distant galaxies and IBHs when using this instrument. This could, perhaps, be done with help of observations at different frequencies and using different variability patterns of IBHs and background sources. It is worth noting that JWST operates at higher frequencies than MM does and, therefore, possesses a much better spatial resolution and is, hence, free from limitations imposed by the confusion effect. Taking into account that surveys of CMZ by these instruments are expected in any case we conclude that they will be able to find accreting IBHs when their number density is larger than the inverse volume of $\mathrm{CMZ}, \sim 10^{-6} \mathrm{pc}^{-3}$, provided that our spectral model is valid and that $\lambda$ is large enough, $\lambda \sim 0.1-1$.

On the other hand, when the number of IBHs within CMZ, $N_{I B H}$, is large, there could be black holes travelling with respect to interstellar medium with velocities smaller than $\sigma$. Assuming the Maxwell statistics of velocity distribution we find that in this case it could be possible to detect IBHs with the accretion efficiency $\lambda \sim 6 / N_{I B H}$ depending on their typical mass as well as IBHs with masses down to $1 \mathrm{M}_{\odot}$.

Our paper is organised as follows. In the next Section we introduce our simplified model of the emission of synchrotron radiation and compare the obtained spectra with those of (Ipser \& Price 1982). In Section 3 we find limitations on black hole masses $M$ and efficiency parameter $\lambda$ from the requirement of detection of an IBH in CMZ by MM and JWST. We would like to stress that even in case of non-detection of IBHs by these instruments it will be possible to obtain limitations on their abundance and/or on the value of $\lambda$. We adopt the cgs system of units throughout the text. Note that Sections 2 and 3 are self-contained, so readers who are interested only in astrophysical applications may start reading the text from Section 3. We discuss our results and related issues in Section 4.

\section{THE SPECTRAL MODEL}

In general, a calculation of the spectrum emerging from an isolated accreting black hole requires an accretion model, which necessarily employs a large number of numerical proce- 
dures. In what follows we would like to try to eliminate as much numerical work as possible and develop the simplest model, which is still able to reproduce quantitatively the numerical results reported in (Ipser \& Price 1982). It turns out that such a model can be constructed when accretion rate is sufficiently small. It employs merely a few straightforward numerical quadratures and not only can easily be used by other researchers but also clarifies the most important physical mechanisms leading to the formation of the emission spectrum.

As we have mentioned, we consider only spherical accretion in this work ${ }^{4}$. Since we are interested only in sub-mm and IR parts of the spectrum, we consider the synchrotron emission, which is the only radiation mechanism responsible for its formation for gas densities, its temperature and values of magnetic field relevant for our systems. We closely follow basic assumptions and notations of (Ipser \& Price 1982) but make a number of additional simplifying assumptions, notably, we consider only a Newtonian problem and treat relativistic effects only in some 'effective' sense, see below.

It is assumed that radial velocity of accreting gas, $v_{r}$, is proportional to the free fall velocity, $v_{r}=-\beta_{V} \sqrt{\frac{2 G M}{R}}$, where $M$ is the black hole mass, $R$ is the radial distance and $\beta_{V}$ is a constant. Gas number density, $n$, can be found from the mass conservation law, $\dot{M}=-4 \pi n v_{r} R^{2}$. Following (Ipser \& Price 1982) we assume that the energy density of magnetic field, $B$, is a fraction of gravitational energy of infalling gas according to relation

$$
\frac{B^{2}}{8 \pi}=\beta_{B} \frac{G M m_{p} n}{R},
$$

where $m_{p}$ is the proton mass and $\beta_{B}$ is another constant factor.

Similar to (Ipser \& Price 1982) we assume that only thermal electrons contribute to the formation of the spectrum. Thus, the emitting electons are distributed over velocities according to the relativistic Maxwellian (or, Maxwell-Juttner) distribution. The intensity of synchrotron emission is very sensitive to electron temperature, $T_{c}$. Unlike (Ipser \& Price 1982) and (Beskin \& Karpov 2005) we do not solve any equations describing a dependency of $T_{e}$ on $R$, postulating that it is a fraction of a 'natural' proton temperature $T_{p r}=\frac{m_{p} c^{2} R_{g}}{k_{B} R}$, where $k_{B}$ is the Boltzmann constant and $R_{g}=\frac{2 G M}{c^{2}}$ is the gravitational radius. Accordingly, we have

$$
T_{e}=\epsilon 10^{12} \frac{1}{r} K
$$

where $r=R / R_{g}$ and $\epsilon \leqslant 1$ is considered to be a constant. Using the known properties of

4 Note that it is straightforward to generalise our approach to the case of radiatively inefficient accretion flows. 
the relativistic Maxwellian distribution and equation (3) we estimate the average Lorentz factor of emitting electrons, $\bar{\gamma}$, as $\bar{\gamma} \approx \frac{3 k_{B} T_{e}}{m_{e} c^{2}} \approx 5.1 \cdot 10^{2} \frac{\epsilon}{r}$.

Using these relations it is easy to find the explicit dependencies of $n$ and $B$ on $r$. We have

$$
n=n_{*} \dot{m} m^{-1} \beta_{V}^{-1} r^{-3 / 2}, \quad n_{*} \approx 2.5 \cdot 10^{10} \mathrm{~cm}^{-3}
$$

and

$$
B=B_{*}\left(\beta_{B} \beta_{V}^{-1} \dot{m} m^{-1}\right)^{1 / 2} r^{-5 / 4}, \quad B_{*} \approx 2.1 \cdot 10^{4} \mathrm{G},
$$

where $m=\frac{M}{10 \mathrm{M}_{\odot}}, \dot{m}=\frac{\dot{M}}{10^{-8} \dot{M}_{E}}$, and $\dot{M}_{E}$ is in turn the Eddington accretion rate calculated for the standard ten per cent radiation efficiency, $\dot{M}_{E} \approx 1.4 \mathrm{~m} \cdot 10^{19} \mathrm{~g} \mathrm{~s}^{-1}$. It is worth noting that (Ipser \& Price 1982) use a quantity $\bar{g}$ related to $\dot{m}$ through $\bar{g}=\frac{1}{16} \beta_{V}^{-1} \dot{m} m^{-1}$.

Luminosity per frequency unit, $L_{\nu}$, due to synchrotron emission reads

$$
L_{\nu}=8 \pi^{2} \int_{R_{\min }}^{\infty} d R^{\prime} R^{\prime 2} e^{-\tau\left(R^{\prime}\right)} j_{\nu}\left(R^{\prime}\right), \quad \tau(R)=\int_{R}^{\infty} d R^{\prime} \frac{j_{\nu}}{B_{\nu}},
$$

where $\nu$ is a given frequency, $j_{\nu}$ is the synchrotron emissivity, $\tau$ is the optical depth, and $B_{\nu}=\frac{2 \nu^{2} k_{B} T}{c^{2}}$ is the Planck intensity in the Rayleigh-Jeans limit.

Expression (6) is a simplified version of that of (Ipser \& Price 1982). The latter contains an additional integration over the angle $\mu$ between the direction to black hole and the direction of photon propagation. Here we imply that the integrand does not depend on this variable and that the integration is performed over the angles larger than $\pi / 2$ assuming that photons with smaller angles are captured by the black hole. This happens close to the radius of the photon sphere around a non-rotating black hole $R_{p h}=1.5 R_{g}$, where the bulk of outgoing radiation is formed. Additionally, the expression for the optical depth is taken over the radial direction contrary to (Ipser \& Price 1982), where it is evaluated along a particular photon orbit, and we neglect all relativistic corrections, effectively taking into account some of them by setting $R_{\text {min }}=R_{p h}$, because radiation from smaller radii is strongly redshifted and must be emitted within a progressively smaller range of angles $\mu$ to escape to infinity as $R$ gets smaller than $R_{p h}$.

Using ((Ipser \& Price 1982)) and equations (3)-(5) it is easy to obtain explicit expressions for all quantities entering (6). We have

$$
j_{\nu}=\frac{3^{1 / 2} e^{3} n B}{8 \pi m_{e} c^{2}} \Phi(x)=j_{*} \beta_{B}^{1 / 2}\left(\beta_{V}^{-1} \dot{m} m^{-}\right)^{1 / 2} \Phi(x), \quad x=\nu / \nu_{c},
$$

where $e$ and $m_{e}$ are the electron charge and mass, respectively, $j_{*}=4.9 \cdot 10^{-9} \mathrm{erg} \mathrm{cm}^{-3} \mathrm{~s}^{-1}$ MNRAS 000, 000-000 (2019) 
and $\nu_{c}$ is the so-called critical frequency

$$
\nu_{c}=\frac{3 e B\left(k_{B} T\right)^{2}}{4 \pi m_{e}^{3} c^{5}}=\nu_{*} \epsilon^{2}\left(\beta_{B} \beta_{V}^{-1} \dot{m} m^{-1}\right)^{1 / 2} r^{-13 / 4}, \nu_{*}=2.5 \cdot 10^{15} \mathrm{~Hz},
$$

where we set a characteristic value of sine of the pitch angle to be equal to unity. $\Phi(x)$ is given by the expression

$$
\Phi(x)=\int_{0}^{\infty} d z z^{2} e^{-z} F\left(x / z^{2}\right), \quad F(x)=x \int_{x}^{\infty} d z K_{5 / 3}(z),
$$

where $K_{5 / 3}(z)$ is a modified Bessel function of the second kind. It is related to the function $\Pi(x)$ introduced in (Pacholczyk 1970) as $\Phi(x)=x \Pi(x)$. The optical depth can be represented as

$$
\tau=\tau^{*}\left(\beta_{B}^{-1} \beta_{V}^{-1} \dot{m} m\right)^{1 / 2} \tilde{\nu}^{-2} \int_{r}^{\infty} d r r^{-7 / 4} \Phi(x), \tau_{*}=7.7 \cdot 10^{-9},
$$

where $\tilde{\nu}=\nu / \nu_{c}(r=1)$.

Substituting (7)-(10) in (6) we obtain

$$
L_{\nu}=L_{*} \beta_{B}^{1 / 2}\left(\beta_{V} \dot{m} m\right)^{3 / 2} \int_{3 / 2}^{\infty} d r r^{-3 / 4} e^{-\tau} \Phi(x), \quad L_{*}=10^{13} \mathrm{erg} \mathrm{s}^{-1} \mathrm{~Hz}^{-1} .
$$

We use expression (11) to compare the results obtained with the help of our simplified model with those of (Ipser \& Price 1982) setting $\beta_{V}=\beta_{B}=1$ as in this paper. After that, the Ipser and Price model depends only on two parameters, which are the black hole mass and their accretion factor $\bar{g}$ proportional to our dimensionless accretion rate $\dot{m}$. The only parameter, which is varied in our model to fit it to that of Ipser and Price is the electron temperature factor $\epsilon$, since the temperature is expected to be smaller than the virial one close to $R_{\min }$. We set $\epsilon=0.2$ to obtain a good agreement between the results corresponding to $m=1$ and $\bar{g}=10^{-4}$ and use other values of $m$ and $\bar{g}$ to validate our model.

The results of comparison are shown in Figs 1-3. As seen from Figs. 1-3, there is a very good agreement between our semi-analytic model and the model of (Ipser \& Price 1982) when $\bar{g}<10^{2}$ for the case of $M=10 \mathrm{M}_{\odot}, \bar{g}<1$ for the case of $M=10^{3} \mathrm{M}_{\odot}$ and $\bar{g}<10^{-2}$ for the case of $M=10^{5} \mathrm{M}_{\odot}$. At larger accretion rates our model overproduces luminosity at sufficiently high frequencies, probably, due to the neglect of synchrotron cooling of electrons. Combining these results we use below the condition that $\frac{\bar{g} M}{10^{3} \mathrm{M}_{\odot}}<1$ as a criterion of validity of our model, which results in the following condition for the mass accretion rate

$$
\dot{m}<1600 \beta_{V} \text {. }
$$

Note, however, that for our purposes it is important to have reliable estimates of radiation flux at frequencies corresponding to the minimal values of MM and JWST sensitivity curves, which are $2.8 \cdot 10^{12} \mathrm{~Hz}$ and $8.6 \cdot 10^{13} \mathrm{~Hz}$, for MM and JWST, respectively, see the next Section. 


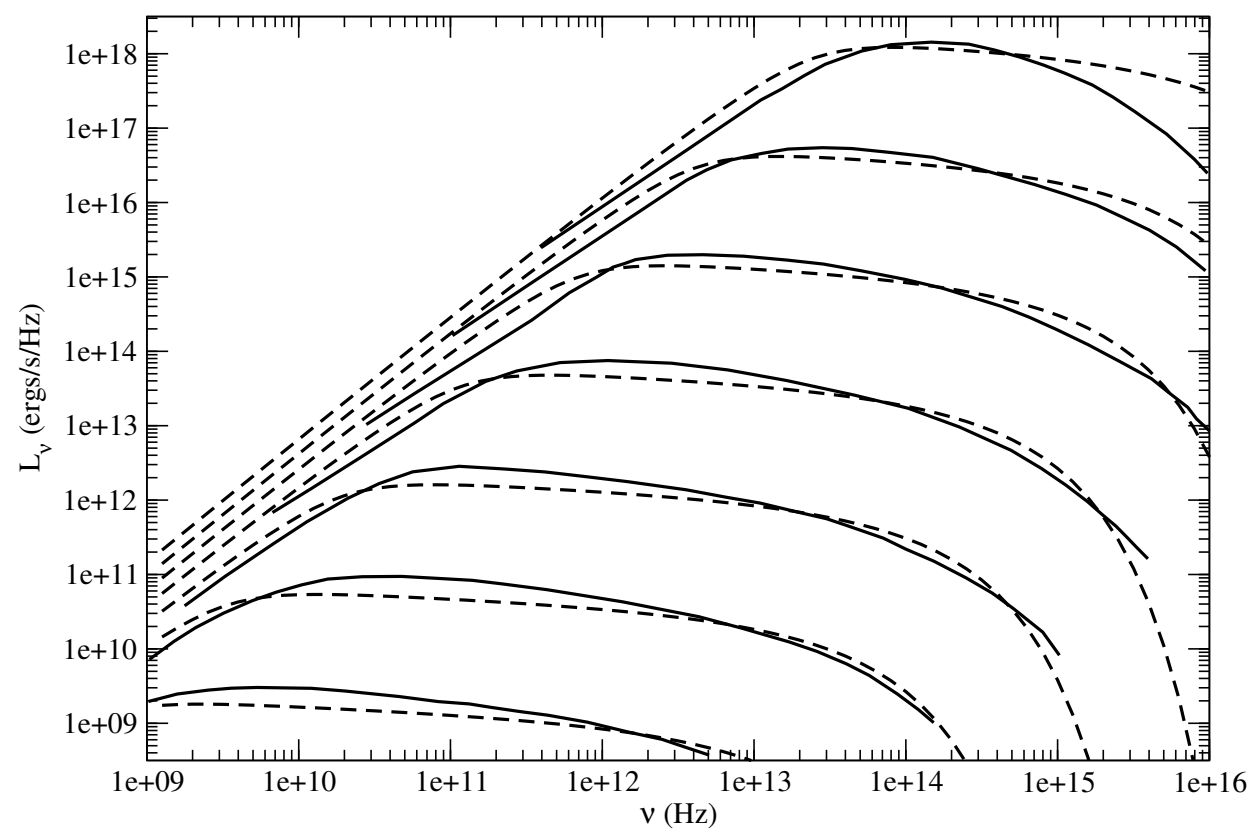

Figure 1. Solid lines reproduce the results of Fig. 5a of (Ipser \& Price 1982), which shows $L_{\nu}$ as a function of $\nu$ calculated in their model for $M=10 \mathrm{M}_{\odot}$ and different values of $\bar{g}$. The corresponding results of our calculations are additionally shown as dashed lines.

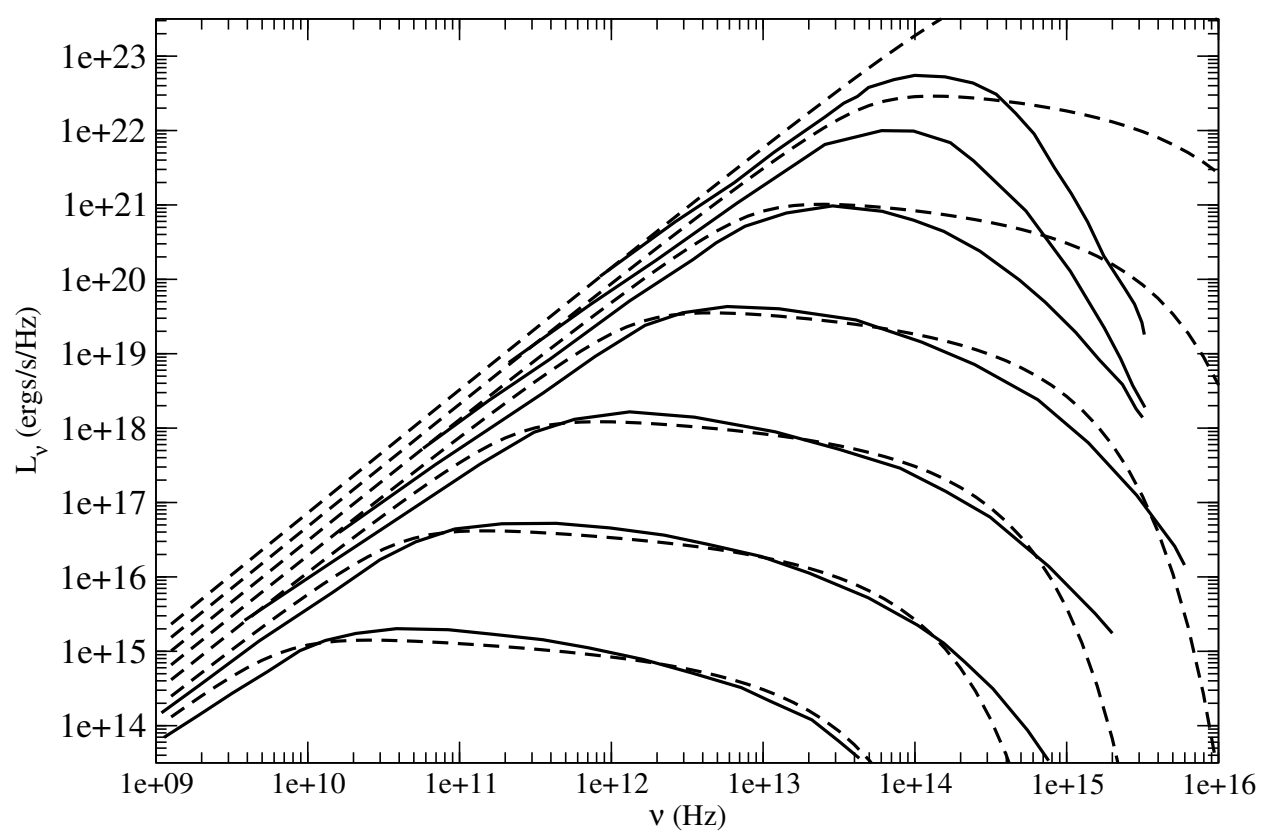

Figure 2. Same as 1, but for $M=10^{3} \mathrm{M}_{\odot}$, see Fig 5b of ((Ipser \& Price 1982)).

As seen from Figs. 1 and 2, at these frequencies the agreement between our model and that of (Ipser \& Price 1982) extends to somewhat larger values of mass flux, so in what follows we are going to imply a less stringent constraint assuming that our model is valid when $\dot{m} \leqslant 10^{4}$.

That our very simple model is able to reproduce results of more complicated numerical 


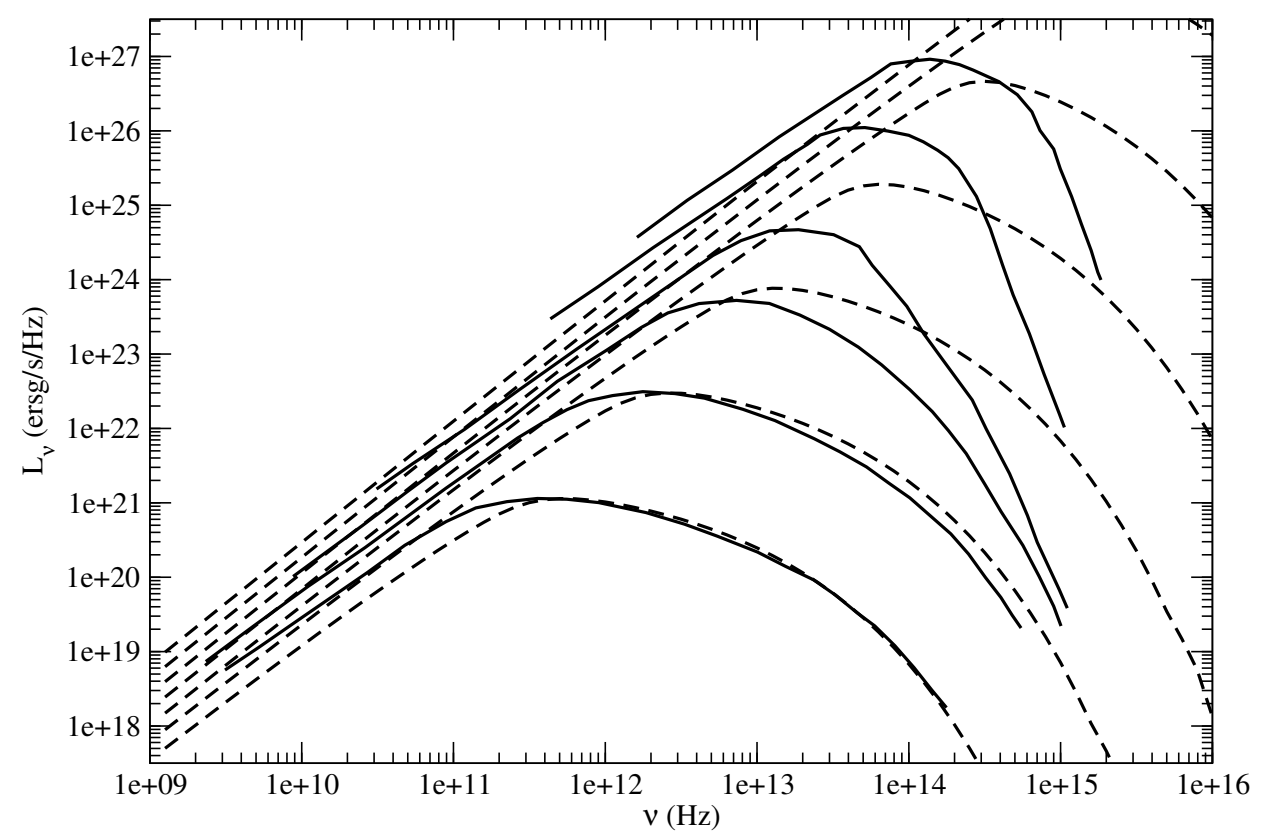

Figure 3. Same as 1, but for $M=10^{5} \mathrm{M}_{\odot}$, see Fig 5c of ((Ipser \& Price 1982)).

calculations implies that the formation of the spectrum rather weakly depends on details of several effects implemented in numerical models, such as e.g. relativistic effects or the dependency of electron temperature on radius, provided that accretion rate is sufficiently small.

\subsection{Semi-analytic expressions for spectrum and luminosity for the case of small optical depth}

When the effects determined by synchrotron optical depth $\tau$ can be neglected, which is always valid at sufficiently high frequencies, one can develop a semi-analytic approach to the calculation of $L_{\nu}$. Moreover, since total luminosity $L_{t o t}=\int d \nu L_{\nu}$ is mainly determined by these high frequencies, we can obtain an approximate analytic expression for it in this limit. For that we neglect the factor $e^{\tau\left(R^{\prime}\right)}$ in the expression for $L_{\nu}$ in (6) and change the integration variable in this expression from $r$ to $x=\nu / \nu_{c}$, see equations (7) and (8). We have

$$
L_{\nu}=\frac{4}{13} L_{0} \tilde{\nu}^{-1 / 13} I(\tilde{\nu}), \quad I(\tilde{\nu})=\int_{\left(\frac{3}{2}\right)^{13 / 4} \tilde{\nu}}^{\infty} d x x^{-12 / 13} \Phi(x),
$$

where $L_{0}=L_{*} \beta_{B}^{1 / 2}\left(\beta_{V} \dot{m} m\right)^{3 / 2}$. The quantity $I(\tilde{\nu})$ can be evaluated numerically, with Fig. 4 showing the result. One can see from this figure that $I(\tilde{\nu})$ decreases monotonically with $\tilde{\nu}$, it is approximately equal to 9 when $\tilde{\nu}=0$ which was checked numerically. Thus, when 


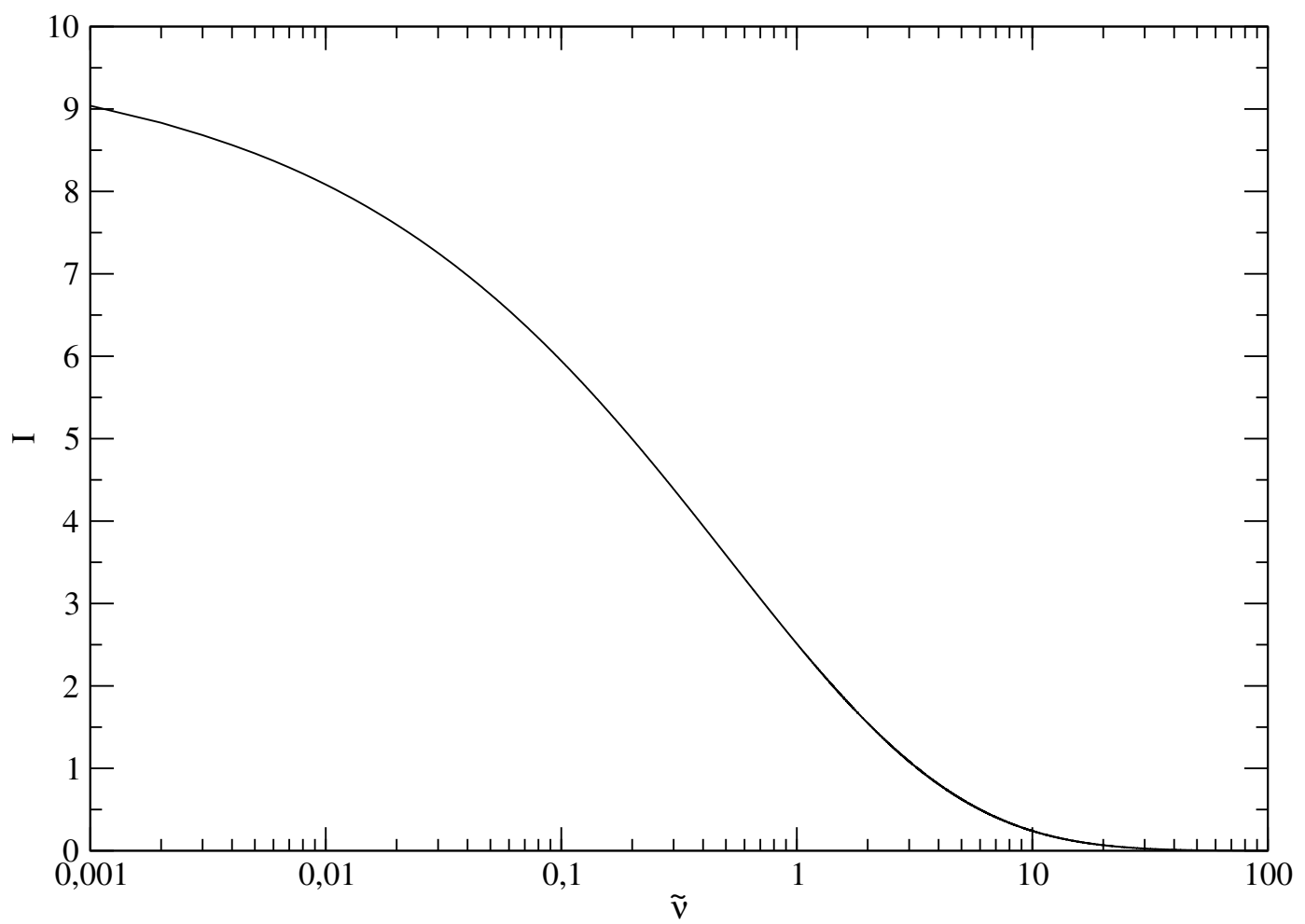

Figure 4. The result of evaluation of the integral $I(\tilde{\nu})$ as a function of the dimensionless frequency $\tilde{\nu}$.

$\tilde{\nu} \ll 1$, but is still sufficiently high for our approximation of zero optical depth to be valid $L_{\nu} \approx \frac{36}{13} L_{0} \tilde{\nu}^{-1 / 13}$, see also e.g. (Bisnovatyi-Kogan \& Ruzmaikin 1976), (Ipser \& Price 1982) and references therein. The total luminosity is given by the expression

$$
L_{t o t}=\frac{4}{13} L_{0} \nu_{0} \int_{0}^{\infty} d \tilde{\nu} \tilde{\nu}^{-1 / 13} I(\tilde{\nu})
$$

where we formally extend integration limits from 0 to $\infty$ and $\nu_{0}=\nu_{c}(r=1)=\nu_{*} \epsilon^{2}\left(\beta_{B} \beta_{V}^{-1} \dot{m} m^{-1}\right)^{1 / 2}$. It can be checked that the integral in (14) approximately equals to 12.4 and we finally obtain

$$
L_{\text {tot }}=9.5 \cdot 10^{28} \epsilon^{2} \beta_{B} \beta_{V} \dot{m}^{2} m \operatorname{erg~s}^{-1}=3.8 \cdot 10^{27} \epsilon_{0.2}^{2} \beta_{B} \beta_{V} \dot{m}^{2} m \operatorname{erg~s}^{-1},
$$

where $\epsilon_{0.2}=\epsilon / 0.2$ and we remind that we use $\epsilon=0.2$ in our calculations. Note that the expression for luminosity (15) is different from that of (Ipser \& Price 1982). This is because (Ipser \& Price 1982) considered the regime of strong synchrotron cooling of electrons, which is realised at accretion rate larger than those considered in this Paper. Therefore, our expression for $L_{t o t}$ and that of (Ipser \& Price 1982) complement each other. 


\section{THE POSSIBILITY OF DETECTION OF IBHS WITH MM AND JWST}

In order to find under which conditions IBHs can be detected by MM and JWST we need to know a typical density of the interstellar medium and a typical velocity of an IBH, which enter equation (1), a typical number of IBHs, $N_{I B H}$, within a region of GC potentially interesting for observations as well as sensitivity curves of both instruments. The latter, in turn, are determined by internal characteristics of these instruments, background radiation of the region and, in case of MM, which has a worse spatial resolution than JWST, by the effect of confusion between radiating IBHs and distant IR galaxies. Note that equation (1) also contains the accretion efficiency parameter $\lambda$, which is treated below as free.

\subsection{Properties of interstellar medium and IBHs population relevant to the problem}

We remind that we consider both possibilities that IBHs are of primordial origin and were born as a result of stellar evolution. In the former case it is reasonable to assume that their density is a fraction, $f$, of the density of dark matter, $\rho_{D M}$. The value of $\rho_{D M}$ at the distance of the order of 100 pc from GC depends significantly on whether its distribution has a core or a cusp in the centre of our Galaxy. If the former assumption is correct $\rho_{D M}^{c o r e} \approx 4 \cdot 10^{-2} \mathrm{M}_{\odot} \mathrm{pc}^{-3}$ (see e.g. Nesti \& Salucci (2013)), while the latter one yields $\rho_{D M}^{\text {cusp }} \approx 1.9 \mathrm{M}_{\odot} \mathrm{pc}^{-3}$ (see e.g. McMillan (2011). Accordingly, we distinguish between $f^{\text {core }}$ and $f^{\text {cusp }}$ noting that in the latter case our results could give stringent constraints on PBHs abundance in case of nondetection. In the case of IBHs born as a result of stellar evolution their possible number densities depends on the value of kick velocity during the supernova explosion and their formation rate. Tsuna et al. (2018) recently investigated such a problem. From their results it follows that for a typical BH mass equal to $10 \mathrm{M}_{\odot}$ the number density is $\sim 10^{-1} \mathrm{pc}^{-3}$ (D. Tsuna, private communication.) and, accordingly, we represent the number density of stellar evolution IBHs in the form $n_{I B H}=f^{S E} 10^{-1} \mathrm{pc}^{-3}$. Note that unlike $f^{\text {core }}$ and $f^{\text {cusp }}$, $f^{S E}$ could be larger than one.

As a model of interstellar medium we employ the model of distribution of molecular hydrogen $\mathrm{H}_{2}$ within the Central Molecular Zone obtained in paper Ferrière et al. (2007), who describe it as a flattened and elongated toroidal structure inclined with respect to the line of sight. The number density of hydrogen is estimated to peak at $150 \mathrm{~cm}^{-3}$ and decays exponentially in vertical and radial direction with typical lengthscales 18 pc and 137 pc, 
respectively. We define the volume of $\mathrm{CMZ}, V_{C M Z}$, as the boundary of the region, where the number density is larger than $50 \mathrm{~cm}^{-3}$ and use the analytical expression from Ferrière et al. (2007) to estimate it. We get $V_{C M Z}=2.7 \cdot 10^{6} \mathrm{pc}^{3}$. As a typical density inside this volume we take $10^{2} \mathrm{~cm}^{-3}$, which results in the mass density $\rho=3.34 \cdot 10^{-22} \mathrm{~g} \mathrm{~cm}^{-3}$ in eq. (1).

We assume that velocity distribution of IBHs is the same as that of the bulge stars at the appropriate distances from GC. In Valenti et al. (2018) it is shown that the distribution of radial velocities can be approximately modelled by the Maxwellian one, with radial dispersion $\sim 130 \mathrm{~km} \mathrm{~s}^{-1}$. The dispersion of absolute values of velocity $v, \sigma$, should be $\sqrt{3}$ times larger, so we use $\sigma=225 \mathrm{~km} \mathrm{~s}^{-1}$. Note that equation (1) also contains the sound speed $c_{s}$. Taking into account that a typical temperature of the $\mathrm{H}_{2}$ component of CMZ is expected to be small, of the order of $100 \mathrm{~K}$, the sound speed $v_{s} \approx 0.5 \mathrm{~km} \mathrm{~s}^{-1}$ is expected to be smaller than the typical velocities of interest and is neglected from now on.

\subsection{The sensitivity curves of MM and JWST}

The sensitivity of JWST was extracted from its exposure time calculator , see https://jwst.etc.stsci.edu/ for NIRcam and MIRI imaging. The sensitivity of MM was computed assuming that it will have a $10 \mathrm{~m}$ mirror with emissivity of 0.05, surface RMS accuracy of 10 microns and temperature $20 \mathrm{~K}$. The sensitivity depends on the level of detector noise, the emission of telescope construction and the sky background. The detectors are characterised by the Noise Equivalent Power $\mathrm{NEP}=10^{-19} \mathrm{~W} \mathrm{~Hz}^{-1 / 2}$. The background sky emission is calculated using the dust model from Planck Collaboration et al. (2014). The dust parameters were averaged over a circle of radius $200 \mathrm{pc}$ around the Galactic centre. The spectral resolution is set to $\lambda / \Delta \lambda=5$.

The fact that our Galaxy is transparent in the far infrared band, with the optical depth below 0.01 even close to its centre, on one hand enhances our ability to search for IBHs in this band, but on the other hand, leads to a serious limitation. The high density of distant submillimetre galaxies on the sky together with relatively low resolution of even a $10-\mathrm{m}$ submillimetre telescope result in the effect of confusion: below a certain level of flux density images of point sources on the sky start to overlap. This critical level of flux density depends on the telescope diameter and on wavelength. For MM it was estimated in Ermash et al. (2018), based on N-body cosmological simulations and a model of galactic emission, and we 
use this estimate in the present work. For wavelengths shorter than 50 microns the confusion by distant galaxies disappears, so it is not important for JWST.

Also, the observations of the GC at wavelengths shorter than 30 microns are affected by extinction. We adopt the infrared extinction towards the GC from Fritz et al. (2011) and take it into account for computing the JWST sensitivity.

One should note that even if we look for a source with a flux density below the confusion limit, it still can be observed if some criterion is used allowing to distinguish it from distant galaxies. Indeed, differences in the spectral energy distribution (IBHs have a power-law SED in submm range) and variability can be used to distinguish IBH candidates from extragalactic sources. Distant galaxies, even if they host an accreting supermassive black hole, should have a plenty of spectral lines in the submillimeter range. These lines can be used to determine the redshift of a galaxy and other parameters, including AGN activity. They also can be used to get rid of the confusion, see e.g. (Raymond et al. 2010). The sensitivity of Millimetron in its spectral mode of observations is sufficient to detect spectral lines of a galaxy with a flux density 2 orders of magnitude below the confusion limit at 300 microns. The small mass of IBHs in comparison with supermassive black holes should result in a rapid variability of the observed flux density. Actively accreting stellar-mass BHs show detectable variations on very large range of frequencies up to $0.1 \mathrm{~Hz}$ in X-rays (Atapin \& Fabrika 2016) and $0.5 \mathrm{~Hz}$ in optical band (Burenin et al. 2011). In the submillimeter range, the SS433 microquasar core shows flux density variations of $20 \%$ on the timescale of 10 minutes. Of course, these variation timescales are determined by processes in an accretion disc, but a spherical accretion onto a black hole of a similar mass should have a comparable variability timescale. This feature could also make it possible to distinguish IBHs from much more massive and, therefore, much more slowly changing AGNs.

Thus, we give all our estimates of IBH detection probability using two variants of MM sensitivity curves: limited by the detector noise and the level of background in this region and limited by the confusion effect.

The detector-limited sensitivity of both JWST and MM depends on time as $t^{-1 / 2}$. We assume that the CMZ will be studied in detail with both these observatories. A typical period of observational time for the key scientific programs of these observatories is about 500-1000 hours. The field of view of JWST NIRcam is $9.7 \mathrm{arcmin}^{2}$, for MIRI $2.3 \mathrm{arcmin}^{2}$, for MM the field of view at highest frequencies will be about $36 \operatorname{arcmin}^{2}$. This means that for 
the imaging of CMZ, a rough estimate of 1 hour integration time seems reasonable within an accuracy of an order of magnitude.

\subsection{Results}

We show the dependencies of luminosities, $\nu L_{\nu}$, calculated in our model as functions of frequency in Figs. 5-7 together with MM and JWST luminosity curves. Figs. 5-7 correspond to $m=0.1,1$ and $10\left(M=1 \mathrm{M}_{\odot}, 10 \mathrm{M}_{\odot}\right.$ and $\left.100 \mathrm{M}_{\odot}\right)$, respectively, with the luminosity curves shown as solid curves, the dashed, dotted and dot dashed curves corresponding to JWST sensitivity limit, MM sensitivity limit with the confusion effect being neglected and that of MM with the confusion effect being taken into account, respectively. The solid curves with larger values correspond to larger $\dot{m}$, every subsequent curve has $\dot{m}$ being multiplied by 10 and we show $\dot{m}$ is the range $10^{-2}-10^{4}$ in case of $m=0.1$ and 1 , see Figs. 5 and 6 , and in the range $10^{-3}-10^{3}$ in case of $m=10$, see Fig. 7 .

One can see from these figures that both instruments can detect IBHs provided that their masses and/or accretion rates are sufficiently large. JWST has a larger range of available $m$ and $\dot{m}$, whereas in the case of MM the confusion effect rather significantly hampers observations and the corresponding range of $m$ and $\dot{m}$ is rather small. We quantify detection capabilities of JWST and MM introducing a critical accretion rate, $\dot{m}_{c}$, above which observations are possible, for a given value of $m$. The dependencies of $\dot{m}_{c}$ on $m$ are shown in Fig. 8, for JWST sensitivity curve and MM sensitivity curves with and without confusion effect as solid, dashed and dotted curves, respectively. This figure demonstrates that in the case of JWST $\dot{m}$ depends on $m$ approximately as a power of $m$. We find that the JWST curve at $0.5 \leqslant m \leqslant 10$ agrees with the expression

$$
\dot{m}_{c}=A m^{-b}, \quad A=2.9, \quad b=0.67
$$

within an order of or less than 10 per cent. This dependency is shown as the dot dashed curve in Fig. $8^{5}$.

The accretion rate given by expression (1) should be smaller that its critical value for $\lambda \leqslant 1$. This requirement sets a limit of the possibility of observations of IBHs by a given

\footnotetext{
${ }^{5}$ It is interesting to point out that a similar expression can be obtained from our approach developed in Section 2.1. Indeed, as seen from Figs. 5-7 a minimal value of JWST sensitivity curve is approximately $5 \cdot 10^{27} \mathrm{ergs} \mathrm{s}^{-1}$, which is close to the numerical value of $L_{t o t}$ given by eq. (15) when $\dot{m}=m=1$. Neglecting factors order of unity and assuming that the total luminosity is mainly determined by frequencies close to that corresponding to the minimum of JWST sensitivity curve, we obtain from (15) an estimate $\dot{m}_{c} \sim m^{-0.5}$, which is quite close to (16).
} 
$[\mathrm{h}]$

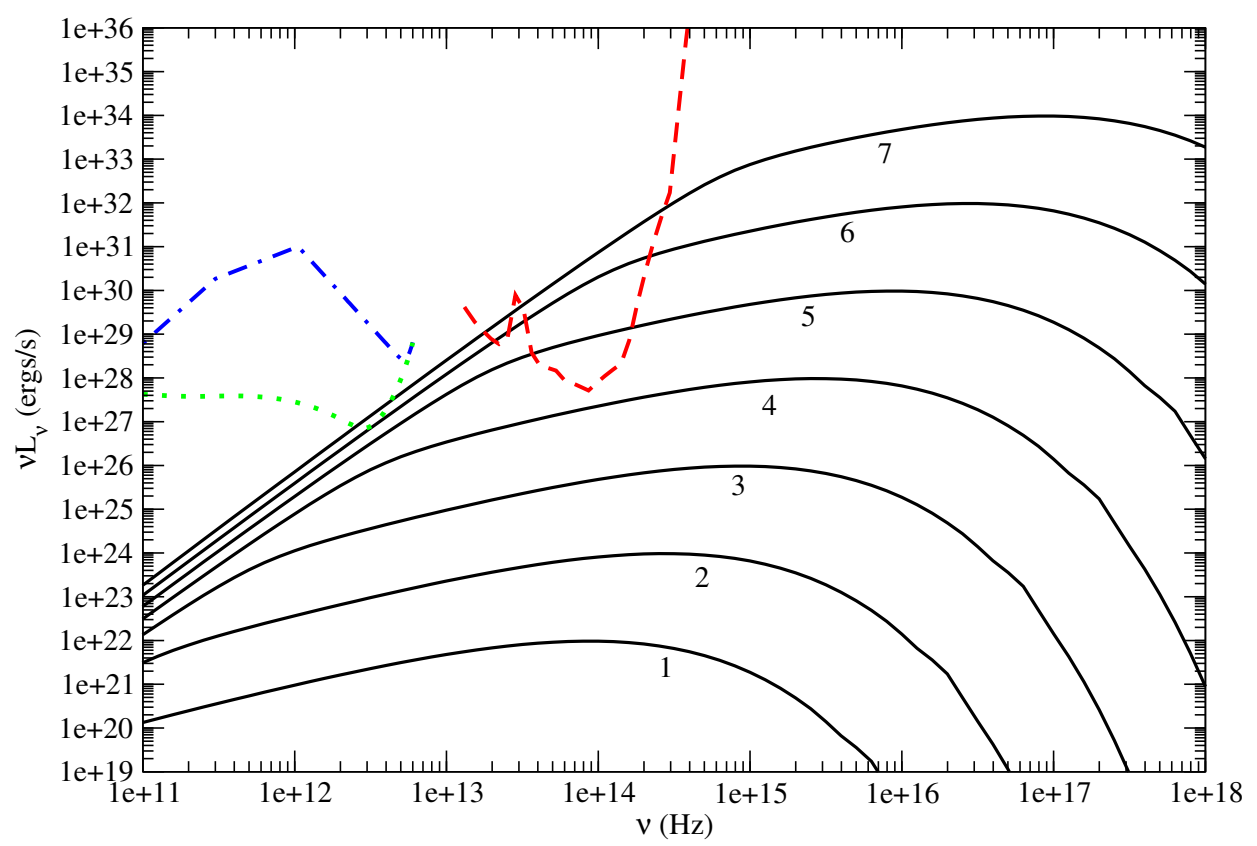

Figure 5. Spectra of an IBH with $M=1 \mathrm{M}_{\odot}$ together with sensitivity curves of MM and JWST. The luminosity curves shown as solid curves, the dashed, dotted and dot dashed curves correspond to JWST sensitivity limit, MM sensitivity limit with the confusion effect being neglected and that of MM with the confusion effect being taken into account, respectively. The solid curves with larger values of their argument correspond to larger $\dot{m}$, the labels $1,2,3,4,5,6$ and 7 denote the curves with $\dot{m}=10^{-2}, 10^{-1}, 1,10,10^{2}, 10^{3}$ and $10^{4}$, respectively.

[h]

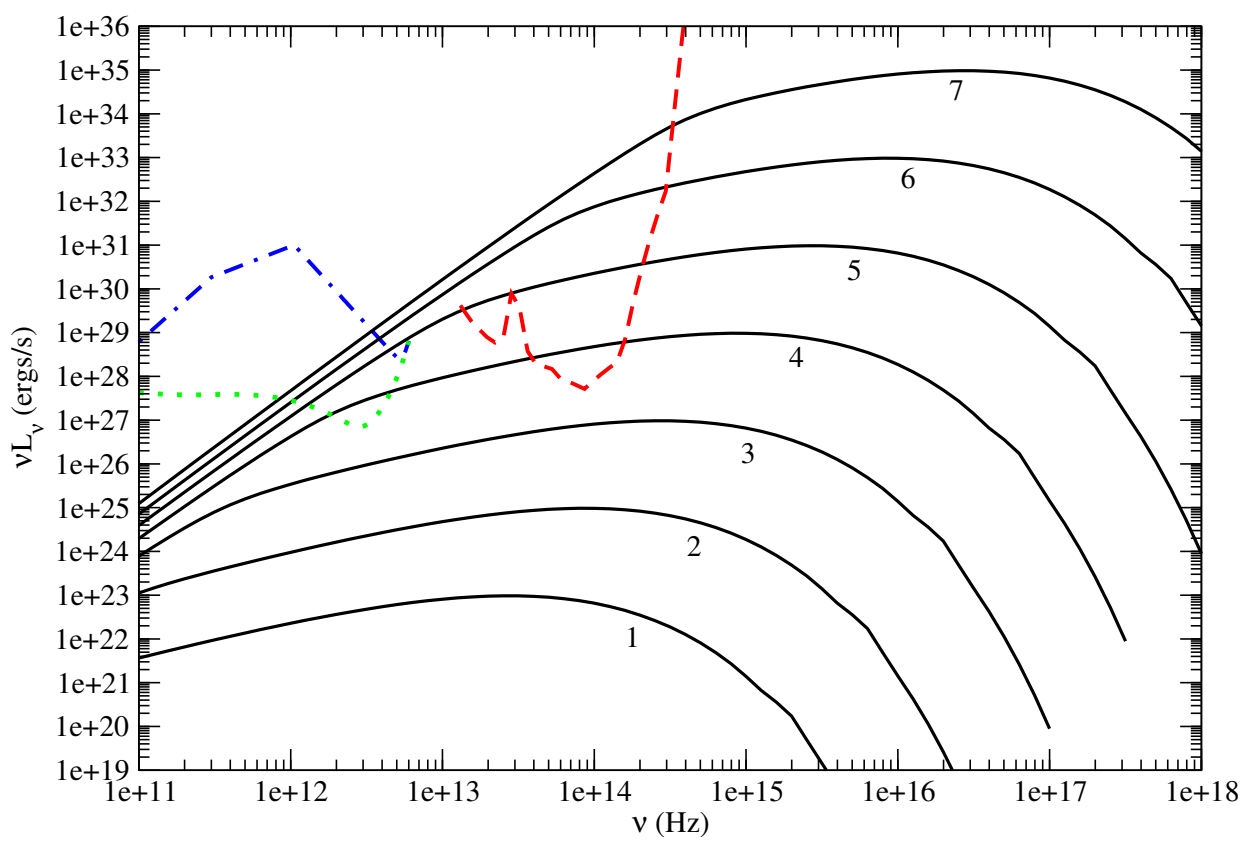

Figure 6. Same as 5, but for $M=10 \mathrm{M}_{\odot}$. 


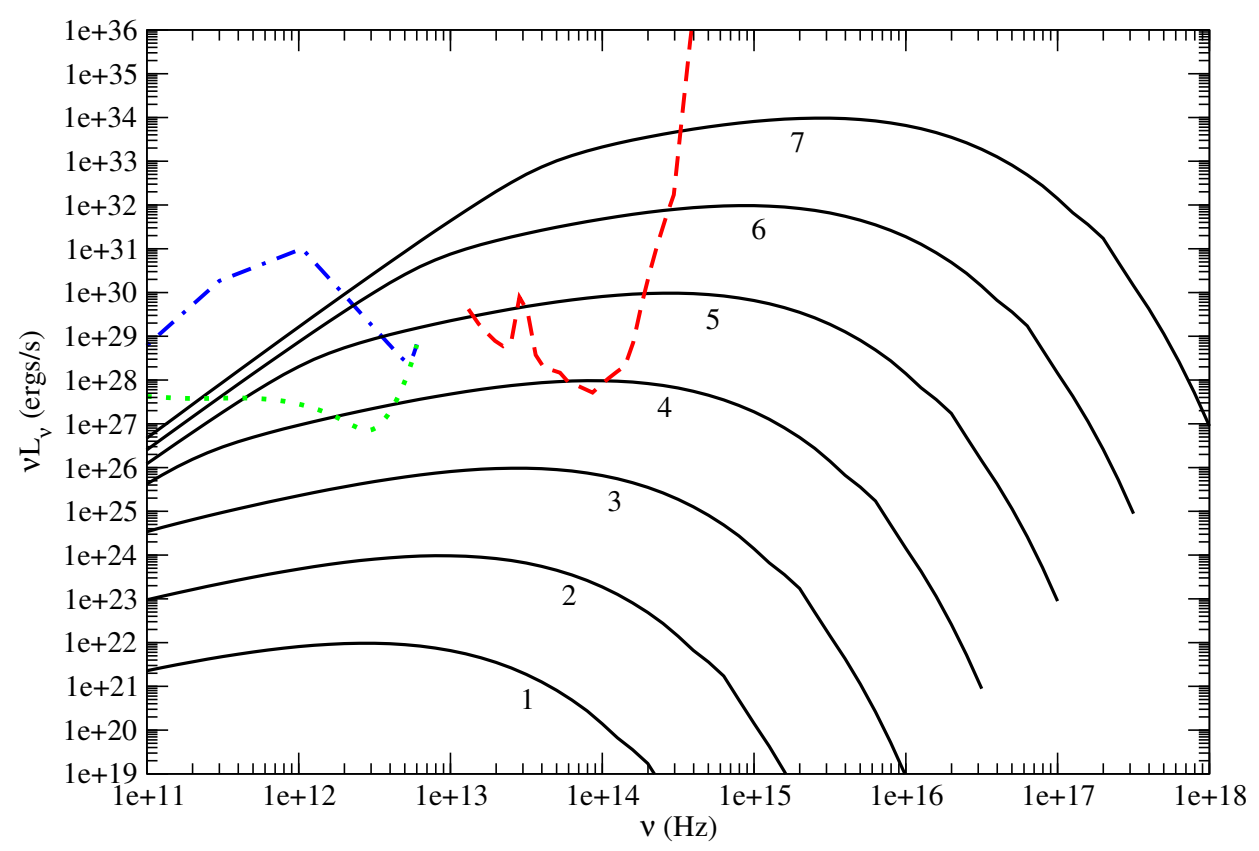

Figure 7. Same as 5 , but for $M=100 \mathrm{M}_{\odot}$. Now we show $\dot{m}$ in the range $10^{-3} 10^{3}$, so the labels $1,2,3,4,5,6$ and 7 denote the curves with $\dot{m}=10^{-3}, 10^{-2}, 1,10,10^{2}, 10^{3}$ and $10^{4}$.

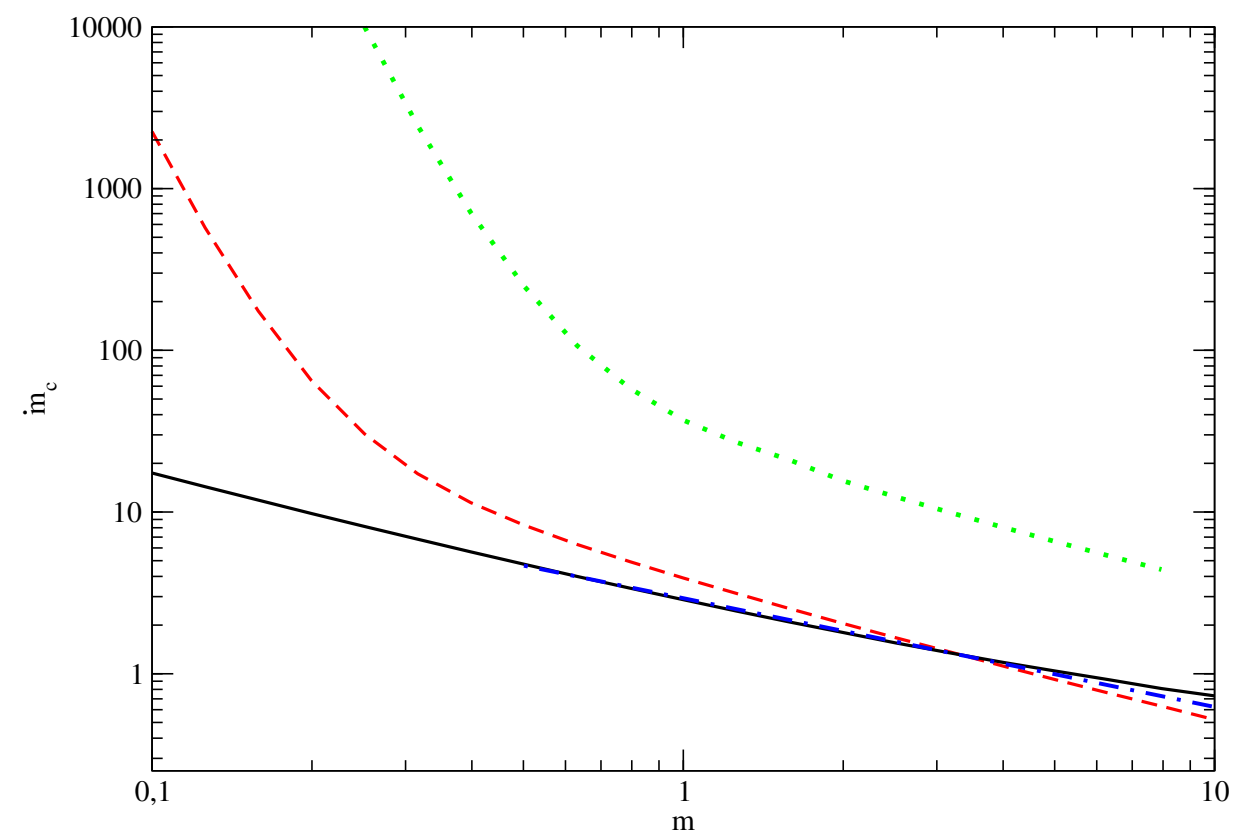

Figure 8. The dependency of dimensionless critical accretion rate $\dot{m}_{c}$ on IBH's dimensionless mass $m=M /\left(10 M_{\odot}\right)$. Solid, dashed and dotted curves correspond to JWST detection limit, that of MM without the confusion effect being taken into account, and that of MM with this effect being accounted, respectively.

instrument and for a given value of the density of the interstellar medium, IBH mass and velocity. In order to compare (1) with $\dot{m}_{c}$, we express the accretion rate given by (1) in terms of our characteristic unit: $\dot{m}=\dot{M} /\left(10^{-8} \dot{M}_{E}\right)$, set $c_{s}=0$ in (1), substitute there our chosen value of density of interstellar medium $\rho=3.34 \cdot 10^{-22} \mathrm{~g} \mathrm{~cm}^{-3}$ and assume that a 'typical' 


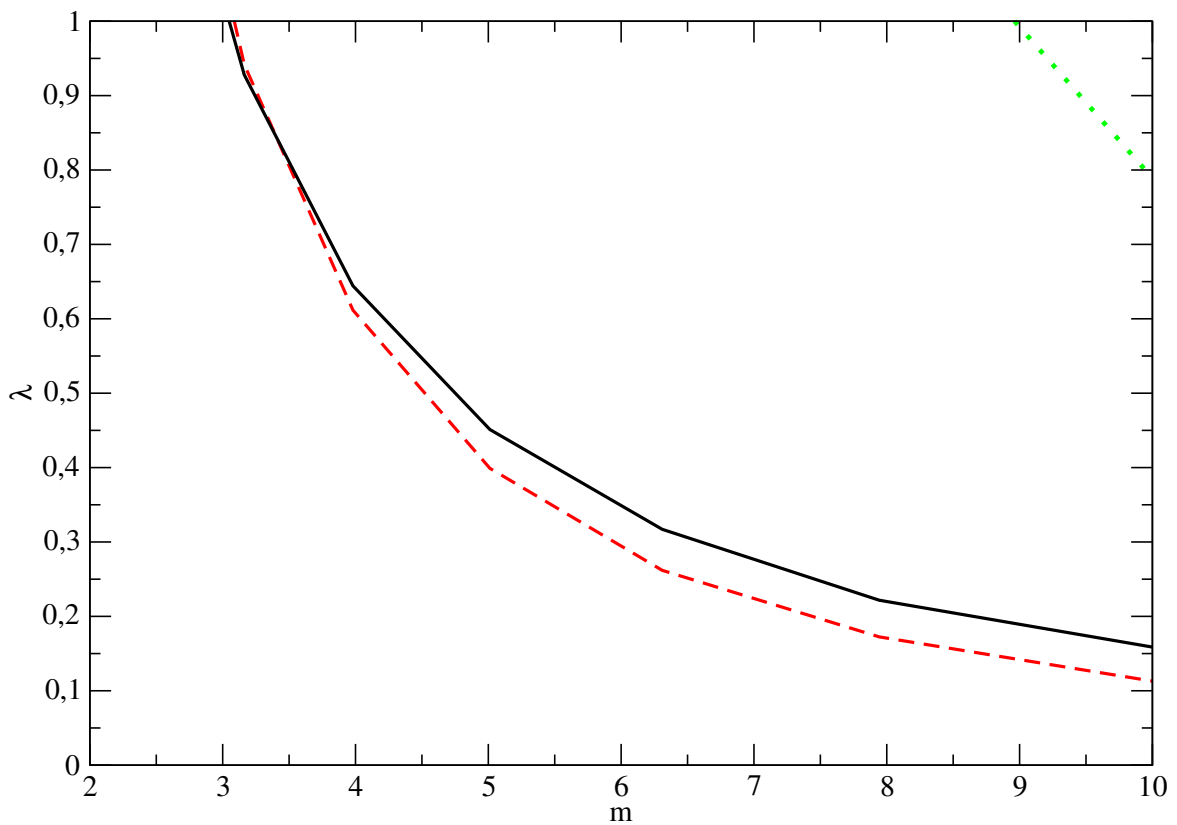

Figure 9. The dependency of the parameter $\lambda$ on $m=M /\left(10 M_{\odot}\right)$. Curves of different styles describe the same cases as in Fig. 8.

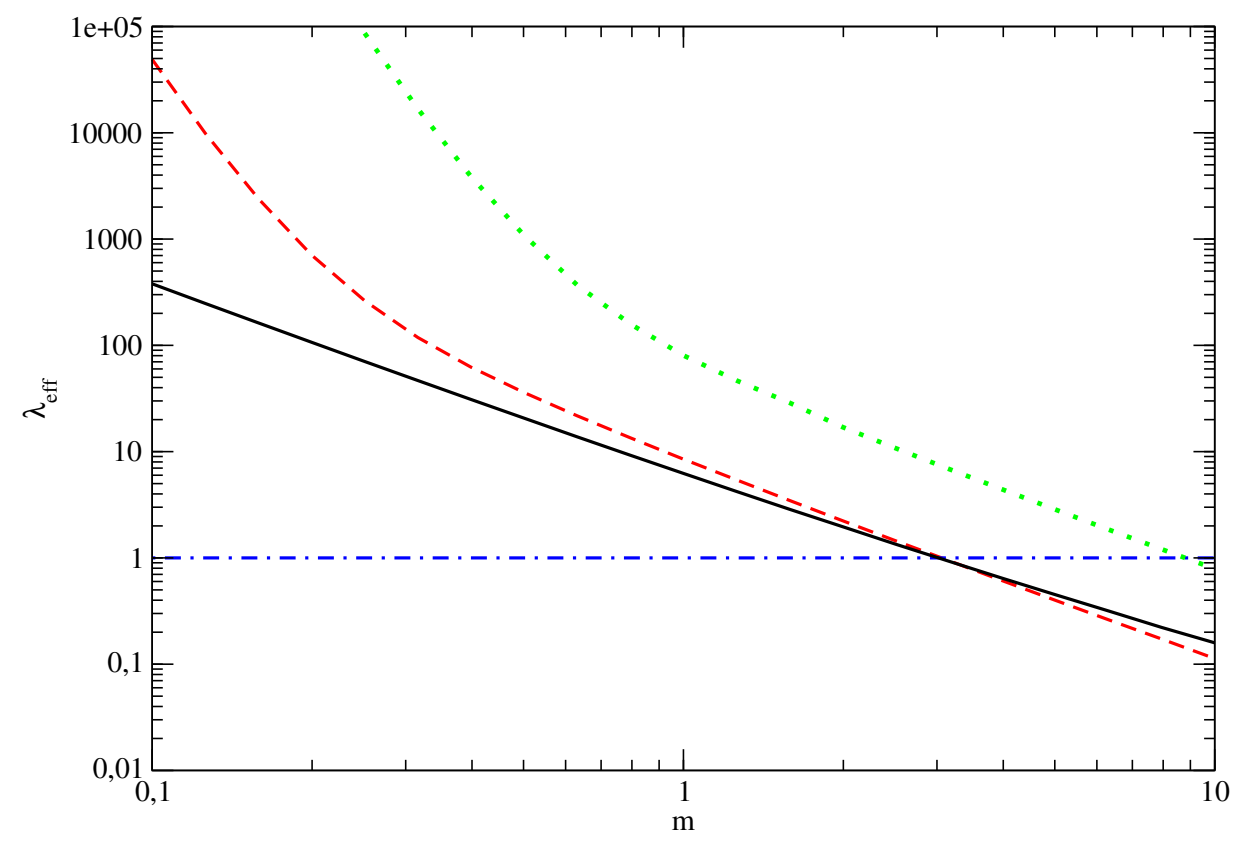

Figure 10. Same as Fig. 9, but now the dependency of parameter $\lambda_{e f f}$ is shown.

IBH velocity $\sigma=225 \mathrm{~km} \mathrm{~s}^{-1}$. We obtain

$$
\dot{m}=0.46 \lambda m \tilde{v}^{-3},
$$

where $\tilde{v}=v / \sigma$.

To begin with let us consider a black hole travelling at the typical velocity and set $\tilde{v}=1$ in eq. (17). In this case from the requirement that $\dot{m}>\dot{m}_{c}$ for $\lambda<1$ we get lower limits on 


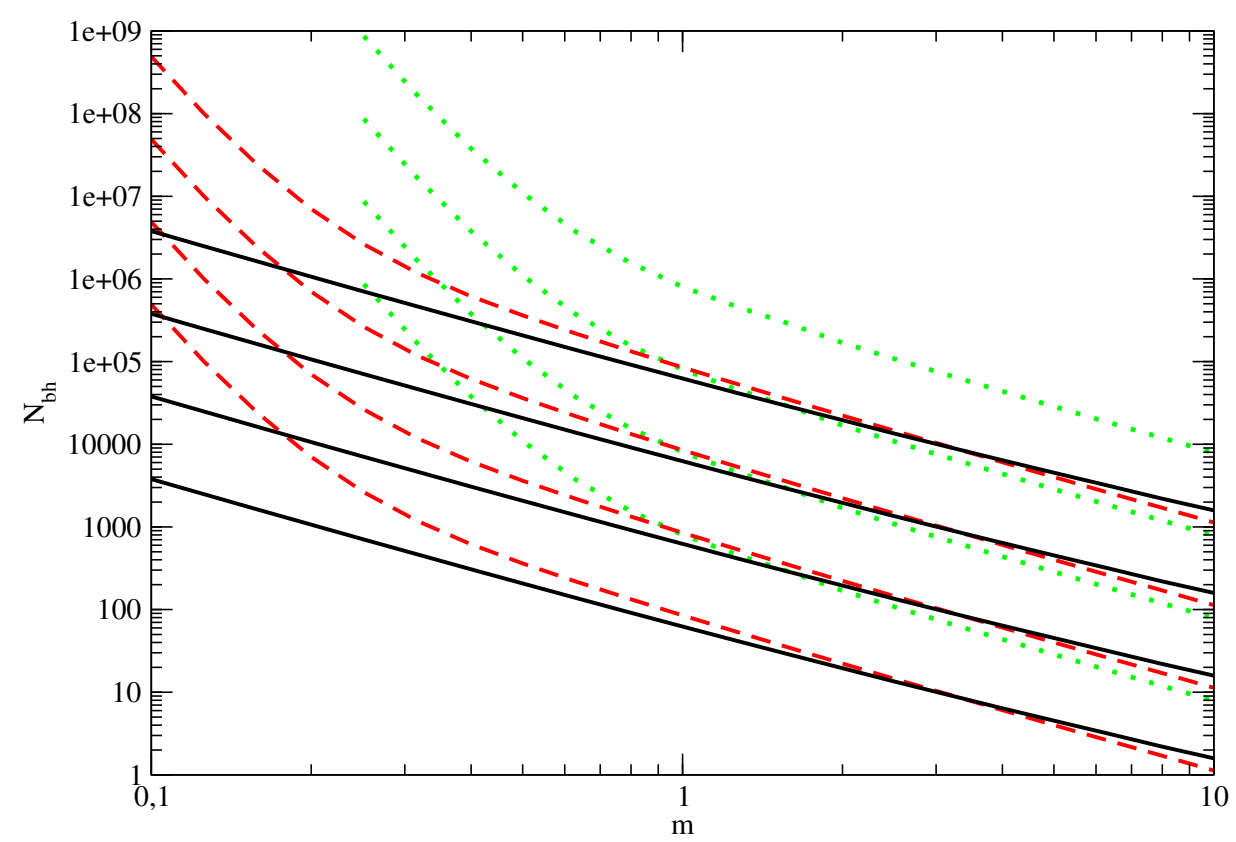

Figure 11. The minimal number of black holes within CMZ needed for observations with JWST and MM, for fixed values of the efficiency parameter $\lambda$, as a function of mass. Solid, dashed and dotted curves corresponding to observations with JWST and MM without and with the confusion limit being taken into account. Curves with progressively larger values of $N_{b h}$ for a given value of $m$ correspond to progressively smaller values of $\lambda$.

black hole masses, $M_{\text {crit }}$, available for observations. For larger black hole masses the condition $\dot{m}=\dot{m}_{c}$ gives a lower limit on the efficiency parameter $\lambda$, which is shown in Fig. 9. One can see from this figure that JWST and MM without taking into account the confusion effect could be able to observe IBHs with masses larger than $M_{\text {crit }}=30 \mathrm{M}_{\odot}$, while allowing for the confusion effect $\mathrm{MM}$ is able to detect IBHs only when $M>90 \mathrm{M}_{\odot}$, respectively. The range of available values of $\lambda$ is rather limited to $\lambda>\sim 0.1$ even for the largest considered black hole mass $100 \mathrm{M}_{\odot}$ when the confusion effect is neglected and $\lambda>0.8$ in the opposite case. That $\lambda$ should be relatively large may be a problem for observations since some works suggest significantly smaller values, see e.g. Igumenshchev \& Narayan (2002). On the other hand, assuming that high accretion efficiency is sustained and taking into account that the available volume of $\mathrm{CMZ} \sim 10^{6} \mathrm{pc}^{3}$, it could be possible to observe IBHs with number density as small as $10^{-6} \mathrm{pc}^{-3}$.

Available values of $\lambda$ and $M_{\text {crit }}$ can be smaller in a situation when there are many IBHs within CMZ, with their number $N_{b h} \gg 1$ and, accordingly, their number density $\gg 10^{-6} \mathrm{pc}^{-3}$. In this case assuming the Maxwellian statistics of distribution of IBHs over velocities we can estimate the number of IBHs having their velocity smaller than $\tilde{v}, N(\tilde{v})$, as $N(\tilde{v})=\tilde{v}^{3} N_{b h}{ }^{6}$. We need at least one IBH for observations, and, from the condition

6 Note that we assume that IBH velocities are defined with respect to GC and do not take into account that molecular 
$N(\tilde{v})=1$ we estimate its velocity as $\tilde{v} \sim N_{b h}^{-1 / 3}$. Substituting this value to (17) we obtain

$$
\dot{m}=0.46 \lambda_{e f f} m, \quad \lambda_{e f f}=N_{b h} \lambda .
$$

The condition of observability $\dot{m} \geqslant \dot{m}_{c}$ results in the lower limit on $\lambda_{\text {eff }}$, which is shown in Fig. 10. Note that it is technically the same curve as shown in Fig. 9, but now the condition $\lambda_{\text {eff }}<1$ is relaxed and we show the whole considered range of $m$, depicting by the additional dot dashed horizontal line $\lambda_{e f f}=1$ the previous range of values ${ }^{7}$. Now, assuming that $\lambda \sim 1$ we find that JWST would be able to detect an IBH even with $M \sim 1 \mathrm{M}_{\odot}$ provided that $N_{b h}>4 \cdot 10^{2}$ while observations with $\mathrm{MM}$ in this mass range require significantly larger $N_{b h} \sim 10^{4}-10^{5}$.

Let us now consider smaller values of $\lambda$ setting, for definiteness, $\lambda=10^{-1}, 10^{-2}, 10^{-3}$ and $10^{-4}$. Using equation (18) and the condition $\dot{m} \geqslant \dot{m}_{c}$ we obtain the lower limits on the number of $N_{b h}$ within CMZ as functions of $m$, for a given value of $\lambda$ and each observational instrument. We plot the results in Fig. 11 with solid, dashed and dotted curves corresponding to observations with JWST and MM without and with the confusion limit being taken into account. Curves with progressively larger values of $N_{b h}$ for a given value of $m$ correspond to progressively smaller values of $\lambda$.

Expression (16) allows us to obtain an analytic expression for $N_{b h}$ and, accordingly, a lower bound on black holes number density $n_{b h}$ and mass density $\rho_{b h}$ for observations with JWST and $m \geqslant 0.5$. Using (18) we have

$N_{b h} \approx 6.3 \cdot 10^{3} \lambda_{-3}^{-1} m^{-1.67}, \quad n_{b h} \approx 2.4 \cdot 10^{-3} \lambda_{-3}^{-1} m^{-1.67} \mathrm{pc}^{-3}, \quad \rho_{b h} \approx 2.4 \cdot 10^{-2} \lambda_{-3}^{-1} m^{-0.67} \mathrm{M}_{\odot} \mathrm{pc}^{-3}$,

where $\lambda_{-3}=\lambda / 10^{-3}$ and we remind that we have adopted the volume of CMZ to be equal to $2.7 \cdot 10^{6} \mathrm{pc}^{3}$. Recalling our definitions for the fractions of IBHs with respect to the mass density of dark matter in case of core and cusp, $f^{\text {core }}$ and $f^{c u s p}$, respectively, and also the definition of the fraction of IBHs number density to the one expected as a result of stellar evolution, $f^{S E}$, we obtain from eq. (19)

$$
f^{\text {core }} \approx 5.8 \cdot 10^{-1} \lambda_{-3}^{-1} m^{-0.67}, \quad f^{\text {cusp }} \approx 1.2 \cdot 10^{-2} \lambda_{-3}^{-1} m^{-0.67}, \quad f^{S E} \approx 2.4 \cdot 10^{-2} \lambda_{-3}^{-1} m^{-1.67} .
$$

Thus, even when the efficiency of accretion onto a PBH is rather small, $\lambda \sim 10^{-2}$ and there

clouds comprising CMZ have themselves appreciable velocities with respect to that. However, a simple estimate shows that our expression for $\tilde{v}$ remains valid by order of magnitude for the expected values of this velocity, which are smaller than or order of $200 \mathrm{~km} \mathrm{~s}^{-1}$, see e.g. Sofue (2013), Henshaw et al. (2016).

7 Note that we have the obvious restriction $\lambda_{\text {eff }} \leqslant N_{b h}$. 
is a cusp in the distribution of dark matter JWST will be able to detect PBHs if they were responsible for a substantial fraction of LIGO events. Its sensitivity is also sufficient to detect IBHs with the number density expected from the stellar evolution according to modern simulations. The limits get better when $m$ increases and improve dramatically for an efficient mode of accretion with $\lambda \sim 1$.

\section{DISCUSSION}

\subsection{Summary and conclusions}

In this Paper we study the possibility of detection of isolated black holes (IBHs) with stellar masses $M \sim 1-100 \mathrm{M}_{\odot}$ travelling within the so-called Central Molecular Zone (CMZ) at distances $\sim 100-200 \mathrm{pc}$ in the submillimetre and infrared range with the help of two planned space observatories, Millimetron (MM) and James Webb Space Telescope (JWST). We develop a simple model of synchrotron emission of thermal electrons by an accreting black hole in the spherical mode of accretion, which, in general, requires several straightforward numerical integrations and is semi-analytic when the optical thickness with respect to synchrotron self-absorption is negligible. It is checked that this model agrees quantitatively with a more complicated numerical model of Ipser \& Price (1982) for the values of accretion rates and black hole masses of interest. We assume the equipartition condition between magnetic field energy density and potential energy density. Also it is assumed that the accretion rate onto the black hole is a fraction, $\lambda$, of the Bondi-Hoyle-Lyttleton value determined, in general, by the density and sound speed of CMZ gas and velocity of the black hole with respect to it.

We use MM and JWST detector sensitivity curves adding to them the contribution of background emission of CMZ gas and, in the case of MM, consider the effect of confusion between the objects of interest and distant submillimetre galaxies. In case of JWST this effect may be shown to be unimportant.

When $\lambda \sim 1$ both JWST and MM can detect a single accreting black hole within CMZ travelling at typical velocity $\sim 200 \mathrm{~km} \mathrm{~s}^{-1}$ provided that its mass is larger than $30 \mathrm{M}_{\odot}$ and, in case of MM the confusion effect is neglected. When this effect is taken into account MM would be able to observe only rather massive black holes with masses $\sim>90 \mathrm{M}_{\odot}$. Bearing in mind that observations of CMZ must be a part of observational programme of both instruments we conclude that in the case of efficient accretion JWST and MM (in 
case when the confusion effect is eliminated by some additional means, say, using different variability timescale and different spectral energy distribution of IBHs and submillimetre galaxies) would be able to observe a single accreting black hole within CMZ. Thus, when $\lambda \sim 1$ it would be possible to observe a single sufficiently massive IBH within CMZ, and, therefore, a possible number density of IBHs within $\sim 100-200$ pc from Galactic Centre could be as small as the inverse volume of CMZ $V_{C M Z} \sim 10^{-6} \mathrm{pc}^{-3}$ for their detection. This exceeds the corresponding number density required to explain the LIGO event by mergers of IBHs of primordial origin.

If a number of IBHs within CMZ is large, observations are still possible even when $\lambda$ is rather small. This is because velocity distribution of IBHs with respect to CMZ gas is likely to be Maxwellian and there could be IBHs with a small velocity even when the bulk of them has typical velocity of the order of its dispersion value. Since the accretion rate sharply increases with decrease of velocity, there could be an IBH with luminosity exceeding the sensitivity limit.

JWST seems to provide a better opportunity for detection of IBHs than MM. This is determined by two circumstances. Firstly, since JWST has higher observational frequencies, it has a better resolution and is, therefore, free from the confusion effect. Secondly, it is fortunate to have the best sensitivity at frequencies order of $10^{14} \mathrm{~Hz}$, which are close to typical frequencies where an IBH has its maximal luminosity. Therefore, we consider this option for JWST in detail. Our finding are expressed in terms of fractions $f$ of some reference mass/number density of IBHs. We consider the case when IBHs are assumed to be primordial and constitute a fraction of dark matter mass density in the case of its distribution in the form of a core or a cusp denoting the corresponding fractions as $f^{\text {core }}$ and $f^{\text {cusp }}$, respectively. We also consider a possibility when IBHs are formed as a result of stellar evolution introducing a fraction, $f^{S E}$, of their number density in terms of a value expected in a recent model of their distribution in the Galaxy. The corresponding results are summarised in equation (19). From this equation it follows that when $\lambda=10^{-2}$ and $M=10 \mathrm{M}_{\odot}$ we have $f^{c u s p} \sim 10^{-3}$. Thus, observations with JWST could detect IBHs constituting a fraction of dark matter sufficient to explain the LIGO events when $\lambda$ is larger than $10^{-2}$ and dark matter distribution has a cusp. 


\subsection{Efficiency of accretion and limits of applicability of our accretion model}

The value of $\lambda$ is very difficult to estimate because there are many factors, which could influence it. Among them are a degree of turbulence and a typical value of magnetic field of CMZ gas, possible velocity shears and density gradients inside CMZ, etc. Since such estimates must necessarily be done by numerical means the issues of computational resolution, potentially large Mach numbers and the necessity to survey a large parameter space of the problem also come into play $^{8}$. Although at the present time a comprehensive approach to this problem is absent it could be possible to make some preliminary considerations based on numerical studies of some limiting cases. When the medium is assumed to be uniform and unmagnetised numerical simulations suggest that $\lambda$ can be order of unity, see El Mellah \& Casse (2015). This conclusion is presumably valid for black holes travelling at velocities order of $\sigma=225 \mathrm{~km} \mathrm{~s}^{-1}$ much larger than other characteristic values of velocity. These are the sound speed $v_{s} \approx 0.5 \mathrm{~km} \mathrm{~s}^{-1}$, the Alfven speed $v_{A} \sim 1 \mathrm{~km} \mathrm{~s}^{-1}$ calculated for the expected typical value of CMZ magnetic field order of $\sim 10^{-5} \mathrm{G}$ (e.g. Yusef-Zadeh et al. (2013)) and a typical velocity of turbulent motion $v_{\text {turb }} \sim 10 \mathrm{~km} \mathrm{~s}^{-1}$, see e.g. Ginsburg et al. (2016), Shetty et al. (2012). On the other hand, the accretion radius $R_{a c c}=\frac{2 G M}{v^{2}} \approx 5 \cdot 10^{12} \mathrm{~cm}$ for $m=1$ and $v=\sigma$ is smaller than a typical size of density inhomogenities and large scale velocity shear, which is assumed to be equal to $R_{i n h} \sim 1 p c$ from now on, see e.g. Shetty et al. (2012).

IBH velocity gets, however, smaller when we consider the tail of Maxwellian distribution over velocities. Remembering that in this case we estimate $v \sim N_{b h} \sigma$, where $N_{b h}$ is the total number of IBHs in CMZ and using eq. (19) we have $v \approx 16 \lambda_{-3}^{1 / 3} m^{0.53} \mathrm{~km} \mathrm{~s}^{-1}$ and $R_{a c c} \approx 2 \cdot 10^{14}$ when $v=16 \mathrm{~km} \mathrm{~s}^{-1}$ and $m=1$. The obtained value of velocity is already of the order of $v_{\text {turb }}$. Unfortunately, we are not aware of any numerical calculations of accretion onto a black hole moving through a turbulent medium with a velocity of the order of an average velocity of turbulent motion. However, a similar case of accretion onto a stationary black hole with the average value of the turbulent velocity order of the sound speed considered in Burleigh et al. (2017) suggests that in this case $\lambda$ could be much smaller that one, with typical values $\sim 10^{-2}-10^{-3}$, which, however, is already taken into account in (19) and (20), where it is implied that $\lambda \sim 10^{-3}$.

Even when the accretion radius is much smaller than a typical size of inhomogenities

8 Note that in case of very large Mach numbers an analytic approach is also possible, see Bisnovatyi-Kogan et al. (1979). 
it was suggested that even a small density/velocity gradient could significantly influence accretion and lead to formation of an accretion disc, see Illarionov \& Sunyaev (1975). Later this problem was considered numerically, see e.g. Ruffert (1999), where it was shown that, in fact, the amount of angular momentum accreted to small distances due to the presence of these inhomogenities is smaller than the analytic estimate. Also, the accreted angular momentum changes sign with time. Either of these effects could inhibit formation of a disc. Also, note that even if a disc is formed, it must have the properties of the so-called Radiatively Inefficient Accretion Flow, which has many similarities with the spherical mode of accretion considered in this Paper and could be treated by similar means, see e.g. Narayan \& Yi (1994).

\subsection{The possibility of radiation emission in other parts of spectrum in our model}

When the typical accretion rate is moderate, $\dot{m} \sim 1$, in our model the bulk of energy is released in the infrared and visible parts of the spectrum. However, from Figs. 5-7 it follows that when $\dot{m}$ is relatively large, a significant fraction of radiation is emitted in X-rays and can be, in principle, detected by the modern X-ray space observatories such as XMM-Newton and Chandra. Let us estimate the possibility of detection of our sources in X-rays. For that we note that the adopted values for the concentration $n_{H_{2}}=100 \mathrm{~cm}^{-3}$ in $\mathrm{CMZ}$ and its characteristic size $d \sim 100$ pc allow us to estimate the optical depth for an X-ray photon: $\tau=\sigma_{H_{2}} N_{H_{2}}$, where $\sigma_{H_{2}}=4.5 \times 10^{-23} \mathrm{~cm}^{2}(E / 1 \mathrm{keV}), N_{H_{2}} \equiv n_{H_{2}} d \approx 3 \times 10^{23} \mathrm{~cm}^{-2}$, and $E$ is the photon energy. The CMZ becomes optically thin at $E \sim 1 \mathrm{keV}$, and we use this value for further estimates. Current experiments such as XMM-Newton and Chandra can observe sources with fluxes at these energies $\sim 10^{-15} \mathrm{erg}^{-2} \mathrm{~s}^{-1}$, which translates to the

threshold value of the X-ray luminosity $L_{x-\text { ray }}=10^{31} \mathrm{erg} \mathrm{s}^{-1}$. The corresponding accretion rate $\dot{m}_{x-\text { ray }}$ is fairly stable in the whole mass range $1-100 M_{\odot}$, gradually increasing from 700 to 1100. Such high accretion rates reside almost at the boundary of the region of validity of our model (see Eq. (11)) and in any case they are much higher than the accretion rates needed for detection in the sub-mm range $\dot{m}_{c}$. That means that IBHs that can be detected both in (sub)mm and X-ray bands comprise only a small subset of IBHs detected only in sub-mm. The ratio of these subsets is determined by the ratio $\dot{m}_{c} / \dot{m}_{x-\text { ray }}$, and changes from $\frac{1}{70}$ for the lightest considered IBHs $\left.\left(1 M_{\odot}\right)\right)$ to $\frac{1}{2000}$ for the heaviest IBHs with $M=100 M_{\odot}$. 
It is also important to note that radiation in harder parts of the spectrum could originate, in principle, from the processes not considered in the Paper, such as the Compton scattering between synchrotron photons and thermal electrons and magnetic reconnection events leading to formation of a tail of high energy non-thermal electrons. However, the

$y$-parameter defined as $y=\int_{R_{\text {min }}}^{\infty} d R \sigma_{T h} n \frac{k_{B} T_{e}}{m_{e} c^{2}}$, where $\sigma_{T h}$ is the Thomson cross section (Illarionov \& Syunyaev (1972)) is extremely small in our case, order of $\sim 10^{-5}$ telling that the Compton effects may be negligible. The possible contribution of non-thermal electrons was considered in Beskin \& Karpov (2005), who assumed that a fraction order of 0.1 of released potential energy of infalling gas is transferred to relativistic electrons with a power-law distribution over their energy. They obtained rather flat spectra extending from infrared to gamma with a maximum of luminosity at frequencies of the order of $10^{15} \mathrm{~Hz}$, which agrees with our approach.

\subsection{A comparison of our approach to other spectral models, which can be used for the search of IBHs}

In other papers considering similar problems of the search of accreting IBHs either the model of Ipser \& Price (1982) (see, e.g. McDowell (1985)) or some phenomenological spectral models (e.g. Fender et al. (2013), Gaggero et al. (2017)) have mainly been used. The former papers have dealt, however, with different observation sites like nearby molecular clouds in case of McDowell (1985). Additionally, they have not considered the observational capabilities of MM and JWST. The latter papers used phenomenological relations between accretion rate and luminosities in different wave bands established either for binary systems or Active Galactic Nuclei. It is not clear to what extent these relations are valid for IBHs.

In particular, our conclusion that, for typical accretion rates, the maximal luminosity is expected to correspond to the optical and infrared bands seemingly contradicts the results of the recent paper Gaggero et al. (2017) who assume that 30 per cent of radiation is released in X-rays and use the fundamental plane relation to estimate luminosity in the radio band. Note, however, that the approach undertaken in this paper relies on the assumption that radiation forms in an accretion disc with properties similar to those found in much more luminous systems observed so far. In this connection we note that in the model of Beskin \& Karpov (2005) luminosity in the harder part of spectrum appears to be an order of 
magnitude smaller than the region close to the maximum, thus, reconfirming the necessity to pay attention to the optical/infrared band for a search of IBHs.

Also note that synchrotron emission can also arise in the bow shock, created by the supersonically moving BH. This effect was investigated in (Wang \& Loeb 2014) in the particular scenario of intermediate mass black holes freely floating in the Milky Way disk. Fig. 3 of (Wang \& Loeb 2014) can be used to show that this mechanism is sub-dominant in the 1-100 $M_{\odot}$ mass range and the radiation flux from the bow-shock is 5-6 orders of magnitude lower than the sensitivity threshold even in the most optimistic case.

\subsection{Other possible sites for observation of IBHs}

IBHs could also be sought for in other locations, such as e.g. Giant Molecular Clouds (GMCs). Let us estimate the possibility of detection taking as an example the Orion Molecular Complex, Ori A and Ori B, which has its typical density $n \sim 10^{2} \mathrm{~cm}^{-3}$, size $R \sim 10 \mathrm{pc}$, mass $M_{H_{2}}=10^{4} M_{\odot}$, and distance $d=500$ pc, see e.g. Pety et al. (2017). We take into account that the accretion rate scales as

$$
\dot{m} \approx\left(\frac{M}{10 M_{\odot}}\right)\left(\frac{n}{10^{2} \mathrm{~cm}^{3}}\right)\left(\frac{v}{300 \mathrm{~km} / \mathrm{s}}\right)^{3}
$$

and calculate the dependencies of luminosities $\nu L_{\nu}$ on frequency for three typical values of IBH mass using $\dot{m}=1$. We show these dependencies together with the relevant MM and JWST sensitivity curves in Fig. 12. The total number of black holes residing inside the MC

can be estimated as $N_{B H}=3 f^{D M} \frac{10 M_{\odot}}{M_{B H}}$, so it is marginally possible to detect IBHs in the nearby GMC provided that their masses are not too small and they comprise a significant fraction of dark matter. In contrast to the CMZ case IBH candidates could be observed in optics due to much lower extinction in this regions. However, since the typical volume of a $\mathrm{GMC} \sim 10^{3} p c^{3}$ is much smaller than that of $\mathrm{CMZ}$, the latter region appears to be preferable for the search of IBHs.

\subsection{A possible development of our approach}

We assume the simplest possible model of CMZ. However, the region with distance order of $100-200 \mathrm{pc}$ from the centre of our Galaxy is a very complex system. In particular, it contains extended molecular clouds with much larger number densities, order of $10^{4} \mathrm{~cm}^{-3}$. It is of interest to repeat calculations reported in this Paper for a realistic model of CMZ. Obviously, there some other sites to look for IBHs such as giant molecular clouds off GC or 


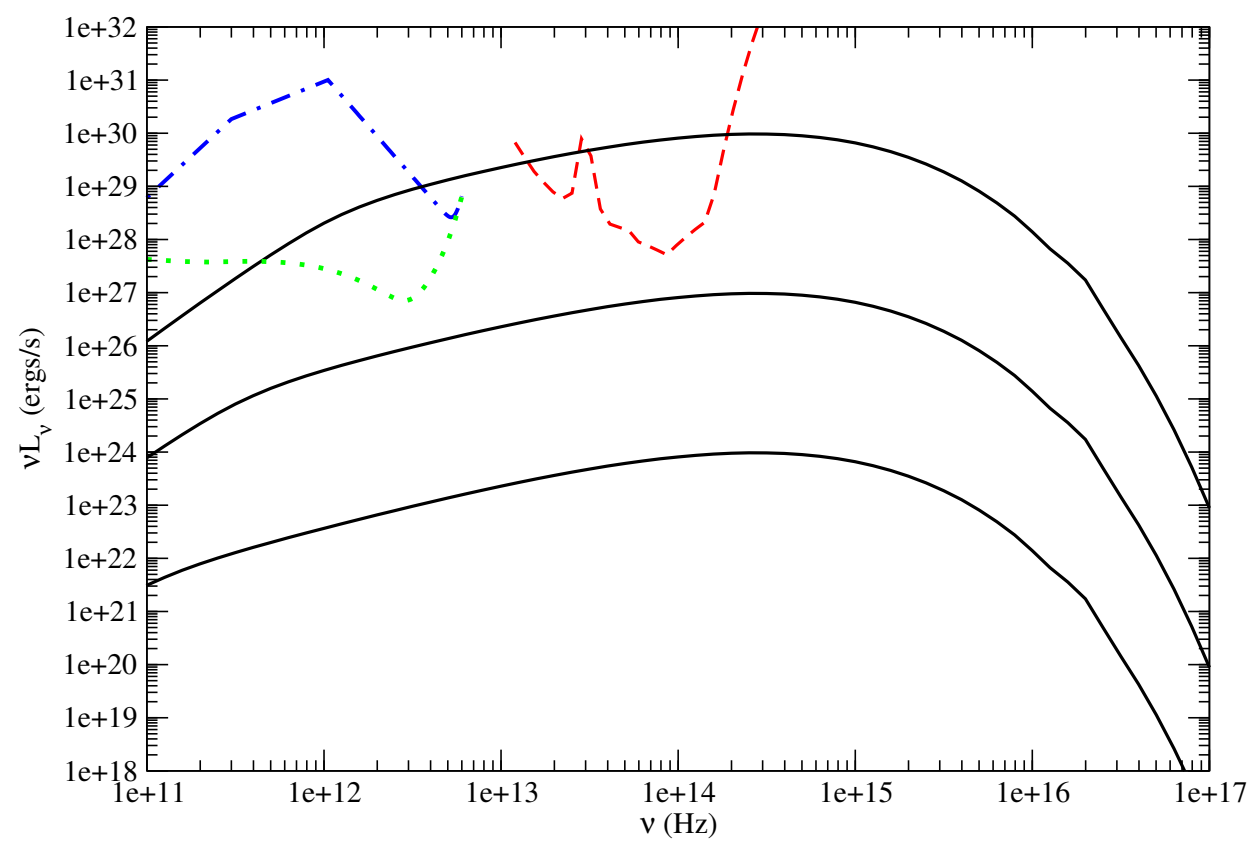

Figure 12. Spectra of an IBHs with $M=1,10$ and $100 \mathrm{M}_{\odot}$ residing in Orion molecular cloud $(d=500$ pc), together with the relevant sensitivity curves of MM and JWST. The luminosity curves are shown as solid curves with larger values of the argument corresponding to larger masses, the dashed, dotted and dot dashed curves correspond to the JWST sensitivity limit, the MM sensitivity limit with the confusion effect being neglected and that of MM with the confusion effect being taken into account, respectively.

some other preferable places. Say, there are models of distribution of compact remnants of stellar evolution in our Galaxy suggesting that they are distributed in a ring of size $5 \mathrm{kpc}$, see Popov \& Prokhorov (1997), Popov \& Prokhorov (1998).

The CMZ is also populated with a prominent population of infrared sources: young stellar objects (YSO). At the distance of CMZ they will look like point sources for both JWST and MM. YSOs possess a large variety of SEDs, some of them may visually look quite similar to those of IBHs (Robitaille et al. 2006; Robitaille 2017). So there is one more issue to be addressed: by future studies how to distinguish them. A more detailed comparison of spectra of YSOs and IBHs can help to answer this question. Finally, the number of faint YSOs in the CMZ is not known. If the number of sources detectable by MM is of the order of $10^{5}$ or higher, they will create the confusion noise, similar to distant galaxies, and the sensitivity of MM may be worse than expected in CMZ. A possible way to suppress the confusion considerably would be to use a difference in patterns of spatial distribution between YSOs and IBHs - while the former reside overwhelmingly inside dense giant molecular clouds, the latter objects are distributed almost uniformly across the CMZ. 


\section{ACKNOWLEDGEMENTS}

We are grateful to E. V. Mikheeva, S. B. Popov and Ya. A. Shchekinov for important discussions and to V. N. Strokov and D. Tsuna for useful comments. This work was supported in part by RFBR grant 17-52-45053 and in part by RFBR grant 19-02-00199. MSP acknowledges support of Leading Science School MSU (Physics of Stars, Relativistic Compact Objects and Galaxies) and support by the Foundation for the Advancement of Theoretical Physics and Mathematics "BASIS" grant 18-1-2-51-1.

\section{REFERENCES}

Agol E., Kamionkowski M., 2002, MNRAS, 334, 553

Armitage P. J., Natarajan P., 1999, ApJ, 523, L7

Atapin K. E., Fabrika S. N., 2016, Astronomy Letters, 42, 517

Bennett D. P., et al., 2002, ApJ, 579, 639

Beskin G. M., Karpov S. V., 2005, A\&A, 440, 223

Bisnovatyi-Kogan G. S., Ruzmaikin A. A., 1976, Ap\&SS, 42, 401

Bisnovatyi-Kogan G. S., Kazhdan Y. M., Klypin A. A., Lutskii A. E., Shakura N. I., 1979, Soviet Ast., 23, 201

Burenin R. A., Revnivtsev M. G., Khamitov I. M., Bikmaev I. F., Nosov A. S., Pavlinsky M. N., Sunyaev R. A., 2011, Astronomy Letters, 37, 100

Burleigh K. J., McKee C. F., Cunningham A. J., Lee A. T., Klein R. I., 2017, MNRAS, 468, 717

Campana S., Pardi M. C., 1993, A\&A, 277, 477

Carr B., Kühnel F., Sandstad M., 2016, Phys. Rev. D, 94, 083504

Chisholm J. R., Dodelson S., Kolb E. W., 2003, ApJ, 596, 437

El Mellah I., Casse F., 2015, MNRAS, 454, 2657

Eldridge J. J., Langer N., Tout C. A., 2011, MNRAS, 414, 3501

Ermash A. A., Pilipenko S. V., Lukash V. N., 2018, arXiv e-prints,

Fender R. P., Maccarone T. J., Heywood I., 2013, MNRAS, 430, 1538

Ferrière K., Gillard W., Jean P., 2007, A\&A, 467, 611

Fritz T. K., et al., 2011, ApJ, 737, 73

Fujita Y., Inoue S., Nakamura T., Manmoto T., Nakamura K. E., 1998, ApJ, 495, L85

Gaggero D., Bertone G., Calore F., Connors R. M. T., Lovell M., Markoff S., Storm E., 2017, Physical Review Letters, 118, 241101

Ginsburg A., et al., 2016, A\&A, 586, A50

Hektor A., Hütsi G., Raidal M., 2018, A\&A, 618, A139

Henshaw J. D., et al., 2016, MNRAS, 457, 2675

Igumenshchev I. V., Narayan R., 2002, ApJ, 566, 137

Illarionov A. F., Sunyaev R. A., 1975, A\&A, 39, 185

Illarionov A. F., Syunyaev R. A., 1972, Soviet Ast., 16, 45

Ipser J. R., Price R. H., 1982, ApJ, 255, 654

Ivanov P. B., Mikheeva E. V., Lukash V. N., Malinovsky A. M., Chernov S. V., Andrianov A. S., Kostenko V. I., Likhachev S. F., 2019, Physics Uspekhi, 62, 1

Kardashev N. S., et al., 2014, Physics Uspekhi, 57, 1199 
Maccarone T. J., 2005, MNRAS, 360, L30

McDowell J., 1985, MNRAS, 217, 77

McMillan P. J., 2011, MNRAS, 414, 2446

Narayan R., Yi I., 1994, ApJ, 428, L13

Nesti F., Salucci P., 2013, J. Cosmology Astropart. Phys., 7, 016

Pacholczyk A. G., 1970, Radio astrophysics. Nonthermal processes in galactic and extragalactic sources

Perna R., Narayan R., Rybicki G., Stella L., Treves A., 2003, ApJ, 594, 936

Pety J., et al., 2017, A\&A, 599, A98

Planck Collaboration et al., 2014, A\&A, 571, A11

Popov S. B., Prokhorov M. E., 1997, Ap\&SS, 252, 351

Popov S. B., Prokhorov M. E., 1998, A\&A, 331, 535

Raymond G., Isaak K. G., Clements D., Rykala A., Pearson C., 2010, PASJ, 62, 697

Robitaille T. P., 2017, A\&A, 600, A11

Robitaille T. P., Whitney B. A., Indebetouw R., Wood K., Denzmore P., 2006, ApJS, 167, 256

Ruffert M., 1999, A\&A, 346, 861

Sasaki M., Suyama T., Tanaka T., Yokoyama S., 2016, Physical Review Letters, 117, 061101

Shapiro S. L., 1973, ApJ, 185, 69

Shapiro S. L., Teukolsky S. A., 1983, Black holes, white dwarfs, and neutron stars: The physics of compact objects

Shetty R., Beaumont C. N., Burton M. G., Kelly B. C., Klessen R. S., 2012, MNRAS, 425, 720

Shvartsman V. F., 1971, Soviet Ast., 15, 377

Sofue Y., 2013, PASJ, 65, 118

Tsuna D., Kawanaka N., Totani T., 2018, MNRAS, 477, 791

Valenti E., et al., 2018, A\&A, 616, A83

Wang X., Loeb A., 2014, Monthly Notices of the Royal Astronomical Society, 441, 809

Yusef-Zadeh F., et al., 2013, ApJ, 762, 33 